\title{
ON STABILITY OF AXIALLY SYMMETRIC SOLUTIONS TO NAVIER-STOKES EQUATIONS IN A CYLINDRICAL DOMAIN AND WITH BOUNDARY SLIP CONDITIONS
}

\author{
M. WIEGNER \\ Department of Mathematics \\ RWTH Aachen, Wuellnerst. 5-7 \\ D-52056 Aachen, Germany \\ W. M. ZAJĄCZKOWSKI \\ Institute of Mathematics \\ Polish Academy of Sciences \\ Śniadeckich 8, 00-956 Warszawa, Poland \\ E-mail:wz@impan.gov.pl \\ and \\ Institute of Mathematics and Cryptology \\ Military University of Technology \\ Kaliskiego 2, 00-908 Warszawa, Poland
}

\begin{abstract}
The existence of global regular axially symmetric solutions to Navier-Stokes equations in a bounded cylinder and for boundary slip conditions is proved. Next, stability of these solutions is shown.
\end{abstract}

1. Introduction. We consider the motion of a viscous incompressible fluid in a bounded cylindrical domain $\Omega \subset \mathbb{R}^{3}$ under boundary slip conditions (see [8])

$$
\begin{array}{ll}
v_{, t}+v \cdot \nabla v-\operatorname{div} \mathbb{T}(v, p)=f & \text { in } \Omega^{T}=\Omega \times(0, T), \\
\operatorname{div} v=0 & \text { in } \Omega^{T}, \\
v \cdot \bar{n}=0 & \text { on } S^{T}=S \times(0, T),
\end{array}
$$

2000 Mathematics Subject Classification: 35Q35, 76D03, 76D05.

Key words and phrases: Navier-Stokes equations, slip boundary conditions, axially symmetric solutions, stability.

Research of W. M. Zajączkowski supported by Polish KBN Grant No 2 P03A 00223.

The paper is in final form and no version of it will be published elsewhere. 


$$
\begin{aligned}
& \bar{n} \cdot \mathbb{T}(v, p) \cdot \bar{\tau}_{\alpha}=0, \quad \alpha=1,2, \quad \text { on } S^{T}, \\
& \left.v\right|_{t=0}=v(0) \quad \text { in } \Omega \text {, }
\end{aligned}
$$

where $v=v(x, t)=\left(v_{1}(x, t), v_{2}(x, t), v_{3}(x, t)\right) \in \mathbb{R}^{3}$ is the velocity vector field, $p=$ $p(x, t) \in \mathbb{R}$ the pressure, $f=f(x, t)=\left(f_{1}(x, t), f_{2}(x, t), f_{3}(x, t)\right) \in \mathbb{R}^{3}$ the external force field, $\bar{n}$ the unit outward vector normal to $S=\partial \Omega, x=\left(x_{1}, x_{2}, x_{3}\right) \in \mathbb{R}^{3}, \bar{\tau}_{1}, \bar{\tau}_{2}$ are unit tangent vectors to $S$ and $\mathbb{T}(v, p)$ is the stress tensor of the form

$$
\mathbb{T}(v, p)=\left\{\nu\left(v_{i, x_{j}}+v_{j, x_{i}}\right)-p \delta_{i j}\right\}_{i, j=1,2,3} \equiv \nu \mathbb{D}(v)-p I,
$$

where $\nu$ is the constant positive viscosity coefficient, $\mathbb{D}(v)$ the dilatation tensor and $I$ the unit matrix.

We introduce the cylindrical coordinates $r, \varphi, z$ by the relations $x_{1}=r \cos \varphi, x_{2}=$ $r \sin \varphi, x_{3}=z$.

By $\Omega$ we denote a cylinder with the $x_{3}$ axis as the axis of symmetry and with boundary $S=S_{1} \cup S_{2}$. By $S_{1}$ we denote the part of the boundary which is parallel to the $x_{3}$ axis and is located at the distance $r=R$ from it. Moreover, it is between the planes $x_{3}=-a$ and $x_{3}=a . S_{2}$ is composed of two parts which are perpendicular to the $x_{3}$ axis and intersect it in two points $x_{3}=-a$ and $x_{3}=a$, respectively. The intersection of $S_{1}$ and $S_{2}$ are two circles which are denoted by $L$.

To introduce axially symmetric solutions we define unit vectors $\bar{e}_{r}=(\cos \varphi, \sin \varphi, 0)$, $\bar{e}_{\varphi}=(-\sin \varphi, \cos \varphi, 0), \bar{e}_{z}=(0,0,1)$, which are directed along the curvilinear coordinates $r, \varphi, z$, respectively. Let $w$ be any vector. Then $w_{r}=w \cdot \bar{e}_{r}, w_{\varphi}=w \cdot \bar{e}_{\varphi}, w_{z}=w \cdot \bar{e}_{z}$.

Definition 1. By an axially symmetric solution to problem (1.1) we mean solution such that $v_{\varphi}=0, v_{r, \varphi}=0, v_{z, \varphi}=0, p_{, \varphi}=0, f_{\varphi}=0, f_{r, \varphi}=0, f_{z, \varphi}=0, v_{\varphi}(0)=0, v_{r, \varphi}(0)=0$, $v_{z, \varphi}(0)=0$.

The aim of this paper is to prove stability of axially symmetric solutions in the sense of [6]. In fact we want to prove existence of global solution to (1.1) which remains close to the axially symmetric solution. For this purpose we formulate a problem defining the axially symmetric solutions and prove their existence in Section 3. To prove the global existence of axially symmetric solutions we use ideas from [3,7], which base on the estimate for vorticity. However, in our case we have different boundary conditions and the estimate for vorticity plays a crucial role.

Having proved global existence of axially symmetric solutions we show its stability by utilizing some ideas from [6]. However our proof is different (see Section 4).

To formulate problems determining an axially symmetric solution to (1.1) we introduce $\Omega_{0}=\left\{x \in \mathbb{R}^{3}: \varphi=0,0<r<R,-a<z<a\right\}, S_{0 i}=\left\{x \in S_{i}: \varphi=0\right\}, i=1,2$, $S_{0}=S_{01} \cup S_{02}$. Then from $[2,4]$ we have

$$
\begin{array}{ll}
v_{r, t}-\nu\left(v_{r, r r}+v_{r, z z}+\frac{1}{r} v_{r, r}-\frac{1}{r^{2}} v_{r}\right)+v_{z} \chi+q_{, r}=f_{r} & \text { in } \Omega_{0}^{T}, \\
v_{z, t}-\nu\left(v_{z, r r}+v_{z, z z}+\frac{1}{r} v_{z, r}\right)-v_{z} \chi+q_{, z}=f_{z} & \text { in } \Omega_{0}^{T},
\end{array}
$$




$$
\begin{array}{ll}
\left(r v_{r}\right)_{, r}+\left(r v_{z}\right)_{, z}=0 & \text { in } \Omega_{0}^{T}, \\
v_{r}=0, \quad v_{z, r}=0 & \text { on } S_{01}^{T}, \\
v_{r, z}=0, \quad v_{z}=0 & \text { on } S_{02}^{T}, \\
\left.v_{r}\right|_{t=0}=v_{r}(0),\left.\quad v_{z}\right|_{t=0}=v_{z}(0), & \text { in } \Omega_{0},
\end{array}
$$

where $\chi=v_{r, z}-v_{z, r}, q=p-\frac{v^{2}}{2}$, and boundary conditions $(1.2)_{4,5}$ are calculated in Lemma 2.1 from $[8$, Ch. 4$]$.

Following $[3,7]$ we formulate the problem for $\chi$ :

$$
\begin{array}{ll}
\chi, t & -\nu\left(\chi_{, r r}+\chi_{, z z}+\frac{1}{r} \chi_{, r}-\frac{1}{r^{2}} \chi\right)+v_{r} \chi, r \\
& \\
-\frac{v_{r}}{r} \chi=v_{z} \chi, z & \\
\chi=0 & \text { in } \Omega_{0}^{T}, \\
\left.\chi\right|_{t=0}=\chi(0) \equiv v_{r}(0)_{, z}-v_{z}(0)_{, r} & \text { on } S_{0}^{T}, \\
& \text { in } \Omega_{0},
\end{array}
$$

where the boundary condition $(1.3)_{2}$ is derived in Lemma 2.2 from [8, Ch. 4].

Since considerations in $[3,7]$ imply that the vorticity problem (1.3) plays a crucial role in the proof of global existence we replace problem (1.1) by problem (1.3), where the velocity is given and the following elliptic problem for $v$ :

$$
\begin{aligned}
& v_{r, z}-v_{z, r}=\chi \quad \text { in } \Omega_{0}, \\
& v_{r, r}+v_{z, z}+\frac{v_{r}}{r}=0 \quad \text { in } \Omega_{0}, \\
& \left.v_{r}\right|_{S_{01}}=0,\left.\quad v_{z}\right|_{S_{02}}=0,
\end{aligned}
$$

where $\chi$ is given.

From Lemma 1.1 from $[8, \mathrm{Ch} .3]$ we know that problems $(1.3),(1.4)$ are equivalent to problem (1.1) if $p$ is a solution to the following problem:

$$
\begin{array}{ll}
\Delta p=-\nabla v \cdot \nabla v+\operatorname{div} f & \text { in } \Omega_{0}, \\
p_{, r}=f_{r} & \text { on } S_{01}, \\
p_{, z}=f_{z} & \text { on } S_{02},
\end{array}
$$

where $v=v_{r} \bar{e}_{r}+v_{z} \bar{e}_{z}$ and $v_{r}, v_{z}$ do not depend on $\varphi$.

Equation $(1.4)_{2}$ implies existence of a function $\psi$ such that

$$
v_{r}=\frac{\psi_{, z}}{r}, \quad v_{z}=-\frac{\psi_{, r}}{r} .
$$

Moreover, boundary conditions $(1.4)_{3}$ give that $\left.\psi_{, r}\right|_{S_{02}}=0$ and $\left.\psi_{, z}\right|_{S_{01}}=0$. Since $\psi$ is defined up to an arbitrary constant we replace problem (1.4) by

$$
\begin{aligned}
& \psi_{, r r}+\psi_{, z z}-\frac{\psi_{, r}}{r}=r \chi \text { in } \Omega_{0}, \\
& \left.\psi\right|_{S_{0}}=0,
\end{aligned}
$$

and $v_{r}, v_{z}$ are determined by (1.6).

Now we look for a stability problem for axially symmetric solutions. Let $v_{a}, p_{a}, f_{a}$ denote the axially symmetric solution to problem (1.1). Then we are looking for solutions 
of (1.1) in the form

$$
v=v_{a}+v^{\prime}, \quad p=p_{a}+p^{\prime}, \quad f=f_{a}+f^{\prime},
$$

where the primed quantities are disturbances which satisfy the problem

$$
\begin{array}{ll}
v_{, t}^{\prime}+v^{\prime} \cdot \nabla v^{\prime}+v^{\prime} \cdot \nabla v_{a}+v_{a} \cdot \nabla v^{\prime}-\operatorname{div} \mathbb{T}\left(v^{\prime}, p^{\prime}\right)=f^{\prime} & \text { in } \Omega^{T}, \\
\operatorname{div} v^{\prime}=0 & \text { in } \Omega^{T}, \\
\bar{n} \cdot v^{\prime}=0 & \text { on } S^{T}, \\
\bar{n} \cdot \mathbb{T}\left(v^{\prime}, p^{\prime}\right) \cdot \bar{\tau}_{\alpha}=0, \quad \alpha=1,2, & \text { on } S^{T}, \\
\left.v^{\prime}\right|_{t=0}=v^{\prime}(0) & \text { in } \Omega .
\end{array}
$$

Now we formulate the main results of this paper.

THEOREM 1 (global existence of axially symmetric solutions). Assume that $v(0) \in$ $L_{2}\left(\Omega_{0}\right), \frac{v_{r}(0)}{r} \in L_{2}\left(\Omega_{0}\right), \frac{\chi(0)}{r} \in L_{3}\left(\Omega_{0}\right), \chi=v_{r, z}-v_{z, r}$,

$$
\begin{aligned}
& \sup _{k}\left[\left\|\frac{f_{r}}{r}\right\|_{L_{2}\left(k T,(k+1) T ; L_{2}\left(\Omega_{0}\right)\right)}+\left\|\frac{F}{r}\right\|_{L_{3}\left(k T,(k+1) T ; L_{3}\left(\Omega_{0}\right)\right)}+\|f\|_{L_{2}\left(k T,(k+1) T ; L_{2}(\Omega)\right)}\right. \\
& \left.+\|\operatorname{div} f\|_{L_{2}\left(k T,(k+1) T ; L_{2}\left(\Omega_{0}\right)\right)}+\|f\|_{L_{2}\left(k T,(k+1) T ; W_{3 / 2}^{1 / 3}\left(\partial \Omega_{0}\right)\right)}\right]<\infty, \\
& F=f_{r, z}-f_{z, r}, \quad k \in \mathbb{N} .
\end{aligned}
$$

Then there exists a global axially symmetric solution to problem (1.1) such that $v \in C\left([k T,(k+1) T] ; H^{1}\left(\Omega_{0}\right)\right) \cap W_{2}^{2,1}\left(\Omega_{0} \times(k T,(k+1) T)\right) \quad$ for any $k \in \mathbb{N}$, which satisfies estimates (3.69) and (3.70).

Let us introduce the quantities

$$
\begin{aligned}
A_{*}(T)= & \sup _{k \in \mathbb{N}}\left(\left\|\bar{f}_{a}\right\|_{L_{2}(k T,(k+1) T)}+\left\|\frac{f_{a r}}{r}\right\|_{L_{2}(\Omega \times(k T,(k+1) T))}+\left\|\operatorname{div} f_{a}\right\|_{L_{2}\left(k T,(k+1) T ; L_{3}(\Omega)\right)}\right. \\
& +\left\|\frac{F_{a}}{r}\right\|_{L_{3}(\Omega \times(k T,(k+1) T)} \\
& \left.+\left\|f_{a}\right\|_{L_{2}\left(k T,(k+1) T ; W_{3 / 2}^{1 / 3}\left(\partial \Omega_{0}\right)\right)}\right) \\
& +\left\|v_{a}(0)\right\|_{L_{2}(\Omega)}+\left\|\frac{\chi_{a}(0)}{r}\right\|_{L_{3}(\Omega)}+\left\|\chi_{a}(0)\right\|_{L_{2}(\Omega)} ; \\
\alpha_{*}(T)= & \frac{1}{\nu^{2}} \sup _{k \in \mathbb{N}}\left\|\frac{f_{a}}{r}\right\|_{L_{2}(\Omega \times(k T,(k+1) T))}+\frac{1}{\nu}\left\|\frac{\chi_{a}(0)}{r}\right\|_{L_{2}(\Omega)}^{2}, \\
D_{*}(T)= & \sup _{k \in \mathbb{N}} e^{\alpha_{*}(T)} \int_{k T}\left[\left\|f(t)-f_{a}(t)\right\|_{L_{2}(\Omega)}^{2}\left[\left.\right|_{\Omega} ^{2}\right] d t+\left\|v(0)-v_{a}(0)\right\|_{L_{2}(\Omega)}^{2} ;\right. \\
& \left.+\left|\int_{\Omega} r\right| v_{\varphi}(t)-\left.\left.v_{a \varphi}(t)\right|^{2} d x\right|^{2}\right]
\end{aligned}
$$




$$
\begin{aligned}
\beta_{*}(T)= & D_{*}(T) \varphi\left(A_{*}(T)\right)+\sup _{k}\left\|f-f_{a}\right\|_{L_{2}(\Omega \times(k T,(k+1) T))} \\
& +\left\|v(0)-v_{a}(0)\right\|_{H^{1}(\Omega)}
\end{aligned}
$$

where $\varphi$ is a polynomial with positive coefficients.

THEOREM 2 (stability). Assume that there exists the axially symmetric solution determined by Theorem 1. Assume that $\sup _{k}\left\|f-f_{a}\right\|_{L_{2}\left(k T,(k+1) T ; L_{2}(\Omega)\right)}<\infty, k \in \mathbb{N}$ $v(0)-v_{a}(0) \in H^{1}(\Omega),\left|\int_{\Omega} r v_{\varphi}(t) d x\right|<\infty$. Assume that $T$ is so large that

$$
e^{-\frac{\nu^{\prime}}{2} T} \leq \frac{1}{2}, \quad \nu^{\prime}<\nu \quad \text { and } \quad \alpha_{*}(T) \leq \frac{\nu^{\prime}}{2} T .
$$

Let there exist a number $\sigma>1$ sufficiently large and $D_{*}(T)$ sufficiently small such that

$$
c\left(\sigma^{5} D_{*}(T) \beta_{*}^{5}(T)+\frac{1}{\sigma}\right) \leq 1,
$$

where $c$ is a constant which depends on the constants from imbedding theorems, estimates of the boundary and on $\nu$. Then $v-v_{a} \in W_{2}^{2,1}(\Omega \times(k T,(k+1) T)), \nabla\left(p-p_{a}\right) \in L_{2}(\Omega \times$ $(k T,(k+1) T))$ for any $k \in \mathbb{N}$ and

$$
\sup _{k}\left[\left\|v-v_{a}\right\|_{W_{2}^{2,1}(\Omega \times(k T,(k+1) T))}+\left\|\nabla\left(p-p_{a}\right)\right\|_{L_{2}(\Omega \times(k T,(k+1) T))}\right] \leq \sigma \beta_{*}(T) .
$$

Stability of axially symmetric solutions, 2d-solutions and helically symmetric solutions is considered in [6]. The authors of [6] assume that the perturbed solution satisfies

$$
\int_{0}^{\infty}\|\nabla v(t)\|_{L_{2}(\Omega)}^{2} d t<\infty .
$$

In this paper we prove the existence of axially symmetric solutions step by step on intervals $[k T,(k+1) T], k \in \mathbb{N}$, so it does not have to vanish with time. Moreover, in [6] there are considered such boundary conditions that the Poincaré inequality holds, which is not used in this paper because the slip boundary conditions are considered. In [5] stability of solutions on 3d-torus is examined. We have to underline that stability in [5] is also considered step by step so (1.10) does not hold.

2. Notation and auxiliary results. Let $u$ be a scalar. Then $|u|$ is the absolute value of $u$. Let $u=\left(u_{1}, u_{2}, u_{3}\right)$ be a vector. Then $|u|=\sqrt{u_{1}^{2}+u_{2}^{2}+u_{3}^{2}}$. By $c$ we denote the generic constant and $c=c(\sigma)$ is an increasing positive function.

To simplify considerations we introduce the notation

$$
\begin{aligned}
& |u|_{p, Q}=\|u\|_{L_{p}(Q)}, \quad Q \in\left\{\Omega, S, \Omega^{T}, S^{T}\right\}, \quad \Omega^{T}=\Omega \times(0, T), \\
& S^{T}=S \times(0, T), \quad p \in[1, \infty] ; \\
& \|u\|_{s, Q}=\|u\|_{H^{s}(Q)}, \quad Q \in\{\Omega, S\}, \quad H^{s}(Q)=W_{2}^{s}(Q), \quad s \in \mathbb{R}_{+} ; \\
& \|u\|_{s, Q^{T}}=\|u\|_{W_{2}^{s, s / 2}\left(Q^{T}\right)}, \quad Q \in\{\Omega, S\}, \quad s \in \mathbb{R}_{+} ; \\
& \|u\|_{s, p, Q}=\|u\|_{W_{p}^{s}(Q)}, \quad\|u\|_{s, p, Q^{T}}=\|u\|_{W_{p}^{s, s / 2}\left(Q^{T}\right)}, \quad Q \in\{\Omega, S\}, \\
& s \in \mathbb{R}_{+}, \quad p \in[1, \infty],
\end{aligned}
$$




$$
|u|_{p, q, \Omega^{T}}=\left(\int_{0}^{T}\left(\int_{\Omega}|u(x, t)|^{p} d x\right)^{q / p} d t\right)^{1 / q}, \quad p, q \in[1, \infty] .
$$

Moreover, we assume that

$$
\begin{aligned}
\|u\|_{L_{p}\left(\Omega_{0}\right)} & =\left(\int_{-a}^{a} \int_{0}^{R}|u(x)|^{p} r d r d z\right)^{1 / p}, \\
\|u\|_{L_{p}(\Omega)} & =\left(\int_{-a}^{a} d z \int_{0}^{R} r d r \int_{0}^{2 \pi} d \varphi|u(x)|^{p}\right)^{1 / p},
\end{aligned}
$$

where $r, \varphi, z$ are the cylindrical coordinates and similarly we define other spaces based on $L_{p}$-spaces.

We use $\bar{u}=\left(u_{r}, u_{z}\right), \bar{\nabla}=\left(\partial_{r}, \partial_{z}\right), \Omega_{0}=\left\{(r, z) \in \mathbb{R}^{2}: 0<r<R,-a<z<z\right\}$, $\Omega_{0 \varepsilon}=\left\{(r, z) \in \mathbb{R}^{2}: 0<\varepsilon<r<R,-a<z<a\right\}$. Moreover, $d x_{0}=r d r d z$.

From [8, Ch. 4] we have the Korn inequality

$$
\left\|v^{\prime}\right\|_{1, \Omega}^{2} \leq c_{1}\left(\left|\mathbb{D}\left(v^{\prime}\right)\right|_{2, \Omega}^{2}+\left|\int_{\Omega} r v_{\varphi}^{\prime} d x\right|^{2}\right) .
$$

To estimate the last term we use the following conservation law which holds for solutions of (1.9) (see [8, Ch. 4, Lemma 2.3])

$$
\int_{\Omega} r v_{\varphi}^{\prime} d x=\int_{\Omega} r v_{\varphi}^{\prime}(0) d x+\int_{0}^{t} d t^{\prime} \int_{\Omega} r f_{\varphi}^{\prime} d x .
$$

For axially symmetric solution we also have the conservation law

$$
\int_{\Omega} r v_{a \varphi} d x=\int_{\Omega} r v_{a \varphi}(0) d x+\int_{0}^{t} d t^{\prime} \int_{\Omega} r f_{a \varphi} d x .
$$

3. Global existence of axially symmetric solutions. In this section we follow the results on global existence of axially symmetric solutions of Ladyzhenskaya [3] and Yudovich-Ukhovskij [7]. Since we are interested in proving global existence of solutions which do not decay with time we consider problem (1.1) step by step in time. Let $k \in \mathbb{N}$ and $T>0$ be given. Then instead of (1.1) we consider the following axially symmetric problem:

$$
\begin{array}{ll}
v_{, t}-\operatorname{div} \mathbb{T}(v, p)=-v \cdot \nabla v+f & \text { in } \Omega_{0} \times(k T,(k+1) T), \\
\operatorname{div} v=0 & \text { in } \Omega_{0} \times(k T,(k+1) T), \\
v \cdot \bar{n}=0, \bar{n} \cdot \mathbb{T}(v, p) \cdot \bar{\tau}_{\alpha}=0, \quad \alpha=1,2, & \text { on } S_{0} \times(k T,(k+1) T), \\
\left.v\right|_{t=0}=v(k T) & \text { in } \Omega_{0} .
\end{array}
$$

Lemma 3.1. Assume that $v(0) \in L_{2}\left(\Omega_{0}\right), f \in L_{2}\left(k T,(k+1) T ; L_{6 / 5}\left(\Omega_{0}\right)\right)$ for any $k \in \mathbb{N}$ and 


$$
B_{1}(T)=\left(\sup _{k} \int_{k T}^{(k+1) T}\left|f\left(t^{\prime}\right)\right|_{6 / 5, \Omega_{0}}^{2} d t^{\prime}\right)^{1 / 2}<\infty .
$$

Assume that $\nu=\nu_{1}+\nu_{2}, \nu_{i} \geq 0, i=1,2$. Then

$$
\begin{aligned}
|\bar{v}(k T)|_{2, \Omega_{0}}^{2} & \leq \frac{c_{1}}{\nu} \frac{B_{1}^{2}(T)}{1-e^{-\nu_{1} T}}+e^{-\nu_{1} k T}|\bar{v}(0)|_{2, \Omega_{0}}^{2} \\
& \leq \frac{c_{1}}{\nu} \frac{B_{1}^{2}(T)}{1-e^{-\nu_{1} T}}+|\bar{v}(0)|_{2, \Omega_{0}}^{2} \equiv A_{1}^{2}(T), \\
|\bar{v}(t)|_{2, \Omega_{0}}^{2} & \leq \frac{c_{1}}{\nu} \int_{k T}^{t}\left|f\left(t^{\prime}\right)\right|_{6 / 5, \Omega_{0}}^{2} d t^{\prime}+|\bar{v}(k T)|_{2, \Omega_{0}}^{2} \leq 2 A_{1}^{2}(T),
\end{aligned}
$$

$$
\int_{k T}^{t}\left(|\bar{\nabla} \bar{v}|_{2, \Omega_{0}}^{2}+\left|\frac{v_{r}}{r}\right|_{2, \Omega_{0}}^{2}\right) d t^{\prime} \leq \frac{1}{\nu}\left(\frac{c_{1}}{\nu} \int_{k T}^{t}\left|f\left(t^{\prime}\right)\right|_{6 / 5, \Omega_{0}}^{2} d t^{\prime}+|\bar{v}(k T)|_{2, \Omega_{0}}^{2}\right) \leq \frac{2}{\nu} A_{1}^{2}(T)
$$

where $t \in[k T,(k+1) T]$.

Proof. From [3] it follows that the axially symmetric solutions to (3.1) satisfy

$$
\frac{d}{d t}|\bar{v}|_{2, \Omega_{0 \varepsilon}}^{2}+\nu\left(|\bar{\nabla} \bar{v}|_{2, \Omega_{0 \varepsilon}}^{2}+\left|\frac{v_{r}}{r}\right|_{2, \Omega_{0 \varepsilon}}^{2}\right) \leq \frac{c_{1}}{\nu}|f|_{6 / 5, \Omega_{0 \varepsilon}}^{2}
$$

where $c_{1}$ is the constant from the imbedding $H^{1}\left(\Omega_{0 \varepsilon}\right) \subset L_{6}\left(\Omega_{0 \varepsilon}\right)$. From (3.5) we have

$$
\frac{d}{d t}\left(|\bar{v}|_{2, \Omega_{0 \varepsilon}}^{2} e^{\nu_{1} t}\right)+\left(\nu_{2}|\bar{\nabla} \bar{v}|_{2, \Omega_{0 \varepsilon}}^{2}+\nu\left|\frac{v_{r}}{r}\right|_{2, \Omega_{0 \varepsilon}}^{2}\right) e^{\nu_{1} t} \leq \frac{c_{1}}{\nu}|f|_{6 / 5, \Omega_{0 \varepsilon}}^{2} e^{\nu_{1} t} .
$$

Integrating (3.6) with respect to time from $k T$ to $t \in[k T,(k+1) T]$ we obtain

$$
\begin{aligned}
& |\bar{v}(t)|_{2, \Omega_{0 \varepsilon}}^{2}+e^{-\nu_{1} t} \int_{k T}^{t}\left(\nu_{2}\left|\bar{\nabla} \bar{v}\left(t^{\prime}\right)\right|_{2, \Omega_{0 \varepsilon}}^{2}+\nu\left|\frac{v_{r}\left(t^{\prime}\right)}{r}\right|_{2, \Omega_{0 \varepsilon}}^{2}\right) e^{\nu_{1} t^{\prime}} d t^{\prime} \\
& \leq \frac{c_{1}}{\nu} e^{-\nu_{1} t} \int_{k T}^{t}\left|f\left(t^{\prime}\right)\right|_{6 / 5, \Omega_{0 \varepsilon}}^{2} e^{\nu_{1} t^{\prime}} d t^{\prime}+|\bar{v}(k T)|_{2, \Omega_{0 \varepsilon}}^{2} e^{-\nu_{1}(t-k T)} .
\end{aligned}
$$

From (3.7) we have

$$
|\bar{v}((k+1) T)|_{2, \Omega_{0 \varepsilon}}^{2} \leq \frac{c_{1}}{\nu} \int_{k T}^{(k+1) T}\left|f\left(t^{\prime}\right)\right|_{6 / 5, \Omega_{0 \varepsilon}}^{2} d t^{\prime}+|\bar{v}(k T)|_{2, \Omega_{0 \varepsilon}}^{2} e^{-\nu_{1}^{\prime} T} .
$$

In view of (3.2) and the inductive considerations inequality (3.8) implies

$$
|\bar{v}(k T)|_{2, \Omega_{0 \varepsilon}}^{2} \leq \frac{c_{1}}{\nu} \frac{B_{1}^{2}(T)}{1-e^{-\nu_{1} T}}+e^{-\nu_{1} k T}|\bar{v}(0)|_{2, \Omega_{0 \varepsilon}} .
$$

Passing with $\varepsilon$ to 0 in (3.9) yields (3.3). Simplifying (3.7) gives

$$
|\bar{v}(t)|_{2, \Omega_{0 \varepsilon}}^{2}+\nu_{2} e^{-\nu_{1} T} \int_{k T}^{t}\left(|\bar{\nabla} \bar{v}|_{2, \Omega_{0 \varepsilon}}^{2}+\left|\frac{v_{r}}{r}\right|_{2, \Omega_{0 \varepsilon}}^{2}\right) d t^{\prime}
$$




$$
\leq \frac{c_{1}}{\nu} \int_{k T}^{t}\left|f\left(t^{\prime}\right)\right|_{6 / 5, \Omega_{0 \varepsilon}}^{2} d t^{\prime}+|\bar{v}(k T)|_{2, \Omega_{0 \varepsilon}}^{2}
$$

where $t \in[k T,(k+1) T]$. Passing with $\varepsilon$ to 0 in inequality (3.10) and inserting $\nu_{1}=0$, $\nu_{2}=\nu$ implies (3.4). This concludes the proof.

LEMma 3.2. Assume that $\frac{\chi(0)}{r} \in L_{2}\left(\Omega_{0}\right), \frac{f}{r} \in L_{2}\left(k T,(k+1) T ; L_{2}\left(\Omega_{0}\right)\right), k \in \mathbb{N}$ and

$$
B_{2}(T)=\left(\sup _{k} \int_{k T}^{(k+1) T}\left|\frac{f(t)}{r}\right|_{2, \Omega_{0}}^{2} d t\right)^{1 / 2}<\infty .
$$

Assume that $\nu=\nu_{1}+\nu_{2}, \nu_{i} \geq 0, i=1,2$. Then

$$
\left|\frac{\chi(k T)}{r}\right|_{2, \Omega_{0}}^{2} \leq \frac{1}{\nu} \frac{B_{2}^{2}(T)}{1-e^{-\nu_{1} T}}+e^{-\nu_{1} k T}\left|\frac{\chi(0)}{r}\right|_{2, \Omega_{0}}^{2} \leq \frac{1}{\nu} \frac{B_{2}^{2}(T)}{1-e^{-\nu_{1} T}}+\left|\frac{\chi(0)}{r}\right|_{2, \Omega_{0}}^{2} \equiv A_{2}^{2}(T),
$$

$$
\begin{aligned}
& \left|\frac{\chi(t)}{r}\right|_{2, \Omega_{0}}^{2} \leq \frac{1}{\nu} \int_{k T}^{t}\left|\frac{f\left(t^{\prime}\right)}{r}\right|_{2, \Omega_{0}}^{2} d t^{\prime}+\left|\frac{\chi(k T)}{r}\right|_{2, \Omega_{0}}^{2} \leq 2 A_{2}^{2}(T), \\
& \int_{k T}^{t}\left|\nabla \frac{\chi\left(t^{\prime}\right)}{r}\right|_{2, \Omega_{0}}^{2} d t^{\prime} \leq \frac{1}{\nu}\left(\frac{1}{\nu} \int_{k T}^{t}\left|\frac{f\left(t^{\prime}\right)}{r}\right|_{2, \Omega_{0}}^{2} d t^{\prime}+\left|\frac{\chi(k T)}{r}\right|_{2, \Omega_{0}}^{2}\right) \leq \frac{2}{\nu} A_{2}^{2}(T),
\end{aligned}
$$

where $t \in[k T,(k+1) T]$.

Proof. From [3] we have

$$
\frac{d}{d t}\left|\frac{\chi}{r}\right|_{2, \Omega_{0 \varepsilon}}^{2}+\nu\left|\nabla \frac{\chi}{r}\right|_{2, \Omega_{0 \varepsilon}}^{2} \leq \frac{1}{\nu}\left|\frac{f}{r}\right|_{2, \Omega_{0 \varepsilon}}^{2},
$$

where $\left|\nabla \frac{\chi}{r}\right|=\left|\bar{\nabla} \frac{\chi}{r}\right|$. From (3.14) we obtain

$$
\frac{d}{d t}\left(\left|\frac{\chi}{r}\right|_{2, \Omega_{0 \varepsilon}}^{2} e^{\nu_{1} t}\right)+\nu_{2}\left|\nabla \frac{\chi}{r}\right|_{2, \Omega_{0 \varepsilon}}^{2} e^{\nu_{1} t} \leq \frac{1}{\nu}\left|\frac{f}{r}\right|_{2, \Omega_{0 \varepsilon}}^{2} e^{\nu_{1} t} .
$$

Integrating (3.15) with respect to time from $k T$ to $t \in[k T,(k+1) T]$ implies

$$
\begin{aligned}
& \left|\frac{\chi(t)}{r}\right|_{2, \Omega_{0 \varepsilon}}^{2}+\nu_{2} e^{-\nu_{1} t} \int_{k T}^{t}\left|\nabla \frac{\chi\left(t^{\prime}\right)}{r}\right|_{2, \Omega_{0 \varepsilon}}^{2} e^{\nu_{1} t^{\prime}} d t^{\prime} \\
& \leq \frac{1}{\nu} e^{-\nu_{1} t} \int_{k T}^{t}\left|\frac{f\left(t^{\prime}\right)}{r}\right|_{2, \Omega_{0 \varepsilon}}^{2} e^{\nu_{1} t^{\prime}} d t^{\prime}+\left|\frac{\chi(k T)}{r}\right|_{2, \Omega_{0 \varepsilon}}^{2} e^{-\nu_{1}(t-k T)} .
\end{aligned}
$$

Inserting $t=(k+1) T$ in (3.16) we get

$$
\left|\frac{\chi((k+1) T)}{r}\right|_{2, \Omega_{0 \varepsilon}}^{2} \leq \frac{1}{\nu} \int_{k T}^{(k+1) T}\left|\frac{f(t)}{r}\right|_{2, \Omega_{0 \varepsilon}}^{2} d t+\left|\frac{\chi(k T)}{r}\right|_{2, \Omega_{0 \varepsilon}}^{2} e^{-\nu_{1} T} .
$$


In view of (3.11) and the inductive considerations inequality (3.17) yields

$$
\left|\frac{\chi(k T)}{r}\right|_{2, \Omega_{0 \varepsilon}}^{2} \leq \frac{1}{\nu} \frac{B_{2}^{2}(T)}{1-e^{-\nu_{1} T}}+e^{-\nu_{1} k T}\left|\frac{\chi(0)}{r}\right|_{2, \Omega_{0 \varepsilon}}^{2} .
$$

Passing with $\varepsilon$ to 0 in (3.18) gives (3.12). Simplifying (3.16) implies

$$
\left|\frac{\chi(t)}{r}\right|_{2, \Omega_{0 \varepsilon}}^{2}+\nu_{2} e^{-\nu_{1} T} \int_{k T}^{t}\left|\nabla \frac{\chi\left(t^{\prime}\right)}{r}\right|_{2, \Omega_{0 \varepsilon}}^{2} d t^{\prime} \leq \frac{1}{\nu} \int_{k T}^{t}\left|\frac{f\left(t^{\prime}\right)}{r}\right|_{2, \Omega_{0 \varepsilon}}^{2} d t^{\prime}+\left|\frac{\chi(k T)}{r}\right|_{2, \Omega_{0 \varepsilon}}^{2}
$$

where $t \in[k T,(k+1) T]$. Passing with $\varepsilon$ to 0 in inequality $(3.19)$ and putting $\nu_{1}=0$, $\nu_{2}=\nu$ yields (3.13). This concludes the proof.

Lemma 3.3. Assume that $\frac{\chi(0)}{r} \in L_{s}\left(\Omega_{0}\right), \frac{F}{r} \in L_{s}\left(k T,(k+1) T ; L_{s}\left(\Omega_{0}\right)\right), s>1, k \in \mathbb{N}$ and

$$
B_{3}(s, T)=\left(\sup _{k} \int_{k T}^{(k+1) T}\left|\frac{F(t)}{r}\right|_{s, \Omega_{0}}^{s} d t\right)^{1 / s}<\infty .
$$

Assume that $\nu=\nu_{1}+\nu_{2}, \nu_{i} \geq 0, i=1,2$. Then

$$
\begin{aligned}
& \left|\frac{\chi(k T)}{r}\right|_{s, \Omega_{0}}^{s} \leq c_{2} \frac{B_{3}^{s}(s, T)}{1-e^{-\nu_{1} T}}+e^{-\nu_{1} k T}\left|\frac{\chi(0)}{r}\right|_{s, \Omega_{0}}^{s} \\
& \leq c_{2} \frac{B_{3}^{s}(s, T)}{1-e^{-\nu_{1} T}}+\left|\frac{\chi(0)}{r}\right|_{s, \Omega_{0}}^{s} \equiv A_{3}^{s}(s, T), \\
& \left|\frac{\chi(t)}{r}\right|_{s, \Omega_{0}}^{s}+\left.\left.\nu \int_{k T}^{t} \int_{\Omega_{0}}|\nabla| \frac{\chi\left(t^{\prime}\right)}{r}\right|^{s / 2}\right|^{2} d x_{0} d t^{\prime} \\
& \leq c_{2} \int_{k T}^{t}\left|\frac{F\left(t^{\prime}\right)}{r}\right|_{s, \Omega_{0}}^{s} d t^{\prime}+\left|\frac{\chi(k T)}{r}\right|_{s, \Omega_{0}}^{s} \leq 2 A_{3}^{s}(s, T),
\end{aligned}
$$

where $t \in[k T,(k+1) T]$.

Proof. From [7] we have the inequality

$$
\frac{d}{d t}\left|\frac{\chi}{r}\right|_{s, \Omega_{0 \varepsilon}}^{s}+\left.\left.\frac{4(s-1)}{s} \nu \int_{\Omega_{0 \varepsilon}}|\nabla| \frac{\chi}{r}\right|^{s / 2}\right|^{2} d x_{0} \leq s \int_{\Omega_{0 \varepsilon}}\left|\frac{F}{r}\right|\left|\frac{\chi}{r}\right|^{s-1} d x_{0} .
$$

Let $s \in[2, \infty)$. Then $(3.23)$ implies

$$
\frac{d}{d t}\left|\frac{\chi}{r}\right|_{s, \Omega_{0 \varepsilon}}^{s}+2 \nu_{1}\left|\frac{\chi}{r}\right|_{s, \Omega_{0 \varepsilon}}^{s}+\left.\left.2 \nu_{2} \int_{\Omega_{0 \varepsilon}}|\nabla| \frac{\chi}{r}\right|^{s / 2}\right|^{2} d x_{0} \leq s \int_{\Omega_{0 \varepsilon}}\left|\frac{F}{r}\right|\left|\frac{\chi}{r}\right|^{s-1} d x_{0} .
$$

We shall perform estimates in (3.24) in two different ways. First we estimate the r.h.s. of (3.24) by

$$
s\left|\frac{F}{r}\right|_{s, \Omega_{0 \varepsilon}}\left|\frac{\chi}{r}\right|_{s, \Omega_{0 \varepsilon}}^{s-1} \leq(s-1) \varepsilon^{\frac{s}{s-1}}\left|\frac{\chi}{r}\right|_{s, \Omega_{0 \varepsilon}}^{s}+\frac{1}{\varepsilon^{s}}\left|\frac{F}{r}\right|_{s, \Omega_{0 \varepsilon}}^{s} \equiv I_{1} .
$$


Assuming that $(s-1) \varepsilon^{\frac{s}{s-1}}=\nu_{1}$ we obtain

$$
I_{1}=\nu_{1}\left|\frac{\chi}{r}\right|_{s, \Omega_{0 \varepsilon}}^{s}+\left(\frac{s-1}{\nu_{1}}\right)^{s-1}\left|\frac{F}{r}\right|_{s, \Omega_{0 \varepsilon}}^{s} .
$$

Then (3.24) takes the form

$$
\frac{d}{d t}\left|\frac{\chi}{r}\right|_{s, \Omega_{0 \varepsilon}}^{s}+\nu_{1}\left|\frac{\chi}{r}\right|_{s, \Omega_{0 \varepsilon}}^{s}+\left.\left.\nu_{2} \int_{\Omega_{0 \varepsilon}}|\nabla| \frac{\chi}{r}\right|^{s / 2}\right|^{2} d x_{0} \leq\left(\frac{s-1}{\nu}\right)^{s-1}\left|\frac{F}{r}\right|_{s, \Omega_{0 \varepsilon}}^{s} .
$$

In the second case we use the imbedding

$$
\left.\left.\int_{\Omega_{0 \varepsilon}}|\nabla| \frac{\chi}{r}\right|^{s / 2}\right|^{2} d x_{0} \geq \frac{1}{c_{1}}\left|\frac{\chi}{r}\right|_{3 s, \Omega_{0 \varepsilon}}^{s},
$$

where $c_{1}$ is the constant from the imbedding $H^{1}\left(\Omega_{0 \varepsilon}\right) \subset L_{6}\left(\Omega_{0 \varepsilon}\right)$. Then the r.h.s. of $(3.24)$ is bounded by

$$
s\left|\frac{F}{r}\right|_{\frac{3 s}{2 s+1}, \Omega_{0 \varepsilon}}\left|\frac{\chi}{r}\right|_{3 s, \Omega_{0 \varepsilon}}^{s-1} \leq(s-1) \varepsilon^{\frac{s}{s-1}}\left|\frac{\chi}{r}\right|_{3 s, \Omega_{0 \varepsilon}}^{s}+\frac{1}{\varepsilon^{s}}\left|\frac{F}{r}\right|_{\frac{3 s}{2 s+1}, \Omega_{0 \varepsilon}}^{s} \equiv I_{2} .
$$

Imposing that $(s-1) \varepsilon^{\frac{s}{s-1}}=\frac{\nu_{2}}{c_{1}}$ we have that

$$
I_{2}=\frac{\nu_{2}}{c_{1}}\left|\frac{\chi}{r}\right|_{3 s, \Omega_{0 \varepsilon}}^{s}+\left(\frac{(s-1) c_{1}}{\nu_{2}}\right)^{s-1}\left|\frac{F}{r}\right|_{\frac{3 s}{2 s+1}, \Omega_{0 \varepsilon}}^{s} .
$$

Then (3.24) takes the form

$$
\left(3.25^{\prime \prime}\right) \frac{d}{d t}\left|\frac{\chi}{r}\right|_{s, \Omega_{0 \varepsilon}}^{s}+2 \nu\left|\frac{\chi}{r}\right|_{s, \Omega_{0 \varepsilon}}^{s}+\left.\left.\nu_{2} \int_{\Omega_{0 \varepsilon}}|\nabla| \frac{\chi}{r}\right|^{s / 2}\right|^{2} d x_{0} \leq\left(\frac{(s-1) c_{1}}{\nu_{2}}\right)^{s-1}\left|\frac{F}{r}\right|_{\frac{3 s}{2 s+1}, \Omega_{0 \varepsilon}}^{s} .
$$

We shall restrict our considerations to the first case. Then $\left(3.25^{\prime}\right)$ implies

$$
\frac{d}{d t}\left(\left|\frac{\chi}{r}\right|_{s, \Omega_{0 \varepsilon}}^{s} e^{\nu_{1} t}\right)+\left.\left.\nu_{2} \int_{\Omega_{0 \varepsilon}}|\nabla| \frac{\chi}{r}\right|^{s / 2}\right|^{2} d x^{\prime} e^{\nu_{1} t} \leq c_{2}\left|\frac{F}{r}\right|_{s, \Omega_{0 \varepsilon}}^{s} e^{\nu_{1} t}
$$

where $c_{2}=\left(\frac{s-1}{\nu}\right)^{s-1}$. Integrating (3.26) with respect to time from $k T$ to $t \in[k T,(k+1) T]$ gives

$$
\begin{aligned}
& \left|\frac{\chi(t)}{r}\right|_{s, \Omega_{0 \varepsilon}}^{s}+\left.\left.\nu_{2} e^{-\nu_{1} t} \int_{k T \Omega_{0 \varepsilon}}^{t}|\nabla| \frac{\chi\left(t^{\prime}\right)}{r}\right|^{s / 2}\right|^{2} d x^{\prime} e^{\nu_{1} t^{\prime}} d t^{\prime} \\
& \leq c_{2} e^{-\nu_{1} t} \int_{k T}^{t}\left|\frac{F\left(t^{\prime}\right)}{r}\right|_{s, \Omega_{0 \varepsilon}}^{s} e^{\nu_{1} t^{\prime}} d t^{\prime}+\left|\frac{\chi(k T)}{r}\right|_{s, \Omega_{0 \varepsilon}}^{s} e^{-\nu_{1}(t-k T)} .
\end{aligned}
$$

From (3.27) we obtain

$$
\left|\frac{\chi((k+1) T)}{r}\right|_{s, \Omega_{0 \varepsilon}}^{s} \leq c_{2} \int_{k T}^{(k+1) T}\left|\frac{F\left(t^{\prime}\right)}{r}\right|_{s, \Omega_{0 \varepsilon}}^{s} d t^{\prime}+\left|\frac{\chi(k T)}{r}\right|_{s, \Omega_{0 \varepsilon}}^{s} e^{-\nu_{1} T} .
$$


In view of (3.20) and inductive considerations we have

$$
\left|\frac{\chi(k T)}{r}\right|_{s, \Omega_{0 \varepsilon}}^{s} \leq c_{2} \frac{B_{3}^{s}(s, T)}{1-e^{-\nu_{1} T}}+e^{-\nu_{1} k T}\left|\frac{\chi(0)}{r}\right|_{s, \Omega_{0 \varepsilon}}^{s} .
$$

Passing with $\varepsilon$ to 0 yields (3.21). Simplifying (3.27) yields

$$
\begin{aligned}
& \left|\frac{\chi(t)}{r}\right|_{s, \Omega_{0 \varepsilon}}^{s}+\left.\left.\nu_{2} e^{-\nu_{1} T} \int_{k T}^{t} \int_{\Omega_{0 \varepsilon}}|\nabla| \frac{\chi\left(t^{\prime}\right)}{r}\right|^{s / 2}\right|^{2} d x_{0} d t^{\prime} \\
& \leq c_{2} \int_{k T}^{t}\left|\frac{F\left(t^{\prime}\right)}{r}\right|_{s, \Omega_{0 \varepsilon}}^{s} d t^{\prime}+\left|\frac{\chi(k T)}{r}\right|_{s, \Omega_{0 \varepsilon}}^{s},
\end{aligned}
$$

where $t \in[k T,(k+1) T]$. Passing with $\varepsilon$ to 0 and inserting $\nu_{1}=0, \nu_{2}=\nu$, inequality (3.30) implies (3.22). This concludes the proof.

Finally we shall obtain estimates for $\frac{v_{r}}{r}$.

LEMMA 3.4. Let the assumptions of Lemma 3.1 and Lemma 3.3 for $s=3$ hold. Let

$$
\begin{aligned}
\sup _{k \in \mathbb{N}}\left(\|\operatorname{div} f\|_{L_{2}\left(k T,(k+1) T ; L_{2}\left(\Omega_{0}\right)\right)}\right. & +\|f\|_{\left.L_{2}(k T,(k+1) T) ; W_{3 / 2}^{1 / 3}\left(\partial \Omega_{0}\right)\right)} \\
& \left.+\left\|\frac{f_{r}}{r}\right\|_{L_{2}\left(k T,(k+1) T ; L_{2}\left(\Omega_{0}\right)\right)}\right)<\infty .
\end{aligned}
$$

Let

$$
\begin{aligned}
& B_{4}^{2}(T)=A_{1}^{2}(T) A_{3}^{2}(3, T)+R^{4} A_{3}^{4}(3, T) \\
& +\sup _{k} \int_{k T}^{(k+1) T}\left(|\operatorname{div} f(t)|_{3, \Omega_{0}}^{2}+\|f(t)\|_{1 / 3,3 / 2, \partial \Omega_{0}}^{2}+\left|\frac{f_{r}(t)}{r}\right|_{2, \Omega_{0}}^{2}\right) d t .
\end{aligned}
$$

Then

$$
\begin{aligned}
& \left|\frac{v_{r}(k T)}{r}\right|_{2, \Omega_{0}}^{2} \leq \frac{c B_{4}^{2}(T)}{1-e^{-\nu_{1} T}}+e^{-\nu_{1} k T}\left|\frac{v_{r}(0)}{r}\right|_{2, \Omega_{0}} \\
& \leq \frac{c B_{4}^{2}(T)}{1-e^{-\nu_{1} T}}+\left|\frac{v_{r}(0)}{r}\right|_{2, \Omega_{0}}^{2} \equiv A_{4}^{2}(T),
\end{aligned}
$$

and

$$
\left|\frac{v_{r}(t)}{r}\right|_{2, \Omega_{0}}^{2}+\nu \int_{k T}^{t}\left|\bar{\nabla} \frac{v_{r}\left(t^{\prime}\right)}{r}\right|_{2, \Omega_{0}}^{2} d t^{\prime} \leq c B_{4}^{2}(T)+\left|\frac{v_{r}(k T)}{r}\right|_{2, \Omega_{0}}^{2} \leq 2 A_{4}^{2}(T) .
$$

Proof. From (1.2) ${ }_{1}$ and boundary conditions we obtain the problem

$$
\begin{array}{lr}
v_{r, t}-\nu\left[\left(r\left(\frac{v_{r}}{r}\right)_{, r}\right)_{, r}+2\left(\frac{v_{r}}{r}\right)_{, r}+v_{r, z z}\right]+v_{z} \chi & \\
=-q_{, r}+f_{r} & \text { in } \Omega_{0 \varepsilon} \times(k T,(k+1) T), \\
\left.v_{r}\right|_{r=R, \varepsilon}=0, & \\
\left.v_{r, z}\right|_{z= \pm a}=0, & \text { in } \Omega_{0 \varepsilon}, \\
\left.v_{r}\right|_{t=k T}=v_{r}(k T) &
\end{array}
$$


where $q=p-\frac{v^{2}}{2}$. Multiplying $(3.34)_{1}$ by $\frac{v_{r}}{r^{2}}$, integrating the result over $\Omega_{0 \varepsilon}$ with the measure $r d r d z$ and using the boundary conditions we get

$$
\begin{aligned}
& \frac{1}{2} \frac{d}{d t}\left|\frac{v_{r}}{r}\right|_{2, \Omega_{0 \varepsilon}}^{2}+\nu\left(\left|\left(\frac{v_{r}}{r}\right)_{, r}\right|_{2, \Omega_{0 \varepsilon}}^{2}+\left|\left(\frac{v_{r}}{r}\right)_{, z}\right|_{2, \Omega_{0 \varepsilon}}^{2}\right) \\
& +\nu \int_{\Omega_{0 \varepsilon}} 2\left(\frac{v_{r}}{r}\right)_{, r} \frac{v_{r}}{r} d r d z=-\int_{\Omega_{0 \varepsilon}} v_{z} \chi \frac{v_{r}}{r} d r d z \\
& -\int_{\Omega_{0 \varepsilon}} q, r \frac{v_{r}}{r} d r d z+\int_{\Omega_{0 \varepsilon}} \frac{f_{r}}{r} \frac{v_{r}}{r} d x_{0} .
\end{aligned}
$$

In view of boundary conditions the last term on the l.h.s. of (3.35) vanishes. The first term on the r.h.s. of (3.35) is estimated by

$$
\varepsilon_{1}\left|\frac{v_{r}}{r}\right|_{6, \Omega_{0 \varepsilon}}^{2}+c\left(1 / \varepsilon_{1}\right)\left|\frac{\chi}{r}\right|_{3, \Omega_{0 \varepsilon}}^{2}\left|v_{z}\right|_{2, \Omega_{0 \varepsilon}}^{2} .
$$

We express the second term on the r.h.s. of (3.35) in the form

$$
-\int_{\Omega_{0 \varepsilon}} p_{, r} \frac{v_{r}}{r} d r d z+\int_{\Omega_{0 \varepsilon}} v v_{, r} \frac{v_{r}}{r} d r d z \equiv I_{1}+I_{2} .
$$

First we examine

$$
I_{1}=-\int_{\Omega_{0 \varepsilon}}(p-\stackrel{\circ}{p})_{, r} \frac{v_{r}}{r} d r d z=\int_{\Omega_{0 \varepsilon}}(p-\stackrel{\circ}{p})\left(\frac{v_{r}}{r}\right)_{, r} d r d z=\int_{\Omega_{0 \varepsilon}} \frac{p-\stackrel{\circ}{p}}{r}\left(\frac{v_{r}}{r}\right)_{, r} d x_{0},
$$

where $\stackrel{\circ}{p}=\left.p\right|_{r=0}$. Continuing, we have

$$
\left|I_{1}\right| \leq \varepsilon_{2}\left|\left(\frac{v_{r}}{r}\right)_{, r}\right|_{2, \Omega_{0 \varepsilon}}^{2}+c\left(1 / \varepsilon_{2}\right)\left|\frac{p-\stackrel{\circ}{r}}{r}\right|_{2, \Omega_{0 \varepsilon}}^{2} .
$$

Next we examine

$$
I_{2}=\frac{1}{2} \int_{\Omega_{0 \varepsilon}}\left(v^{2}\right)_{, r} \frac{v_{r}}{r} d r d z=\frac{1}{2} \int_{\Omega_{0 \varepsilon}}\left(v^{2}-\stackrel{\circ}{v}^{2}\right)_{, r} \frac{v_{r}}{r} d r d z=-\frac{1}{2} \int_{\Omega_{0 \varepsilon}}\left(v^{2}-\stackrel{\circ}{v}^{2}\right)\left(\frac{v_{r}}{r}\right)_{, r} d r d z,
$$

where $\stackrel{\circ}{v}=\left.v\right|_{r=0}$. By the Hölder and Young inequalities we have

$$
\left|I_{2}\right| \leq \varepsilon_{3}\left|\left(\frac{v_{r}}{r}\right)_{, r}\right|_{2, \Omega_{0 \varepsilon}}^{2}+c\left(1 / \varepsilon_{3}\right)\left|\frac{v^{2}-\stackrel{\circ}{v}^{2}}{r}\right|_{2, \Omega_{0 \varepsilon}}^{2},
$$

where the second integral equals

$$
\int_{\Omega_{0 \varepsilon}}\left|\frac{(v-\stackrel{\circ}{v}) \cdot(v+\stackrel{\circ}{v})}{r}\right|^{2} d x_{0} \leq\left|\frac{v-\stackrel{\circ}{v}}{r}\right|_{2 \lambda_{1}, \Omega_{0 \varepsilon}}^{2}|v+\stackrel{\circ}{v}|_{2 \lambda_{2}, \Omega_{0 \varepsilon}}^{2},
$$

where $1 / \lambda_{1}+1 / \lambda_{2}=1$. Finally, we estimate the last term on the r.h.s. of (3.35) by

$$
\varepsilon_{4}\left|\frac{v_{r}}{r}\right|_{2, \Omega_{0 \varepsilon}}^{2}+c\left(1 / \varepsilon_{4}\right)\left|\frac{f_{r}}{r}\right|_{2, \Omega_{0 \varepsilon}}^{2} .
$$


Utilizing the above estimates in (3.35), assuming that $\varepsilon_{1}-\varepsilon_{4}$ are sufficiently small and exploiting the Poincaré inequality we obtain

$$
\begin{aligned}
& \frac{d}{d t}\left|\frac{v_{r}}{r}\right|_{2, \Omega_{0 \varepsilon}}^{2}+\nu\left(\left|\left(\frac{v_{r}}{r}\right)_{, r}\right|_{2, \Omega_{0 \varepsilon}}^{2}+\left|\left(\frac{v_{r}}{r}\right)_{, z}\right|_{2, \Omega_{0 \varepsilon}}^{2}\right) \\
& \leq c\left(\left|\frac{\chi}{r}\right|_{3, \Omega_{0 \varepsilon}}^{2}\left|v_{z}\right|_{2, \Omega_{0 \varepsilon}}^{2}+\left|\frac{p-\stackrel{\circ}{p}}{r}\right|_{2, \Omega_{0 \varepsilon}}^{2}+\left|\frac{v-\stackrel{\circ}{v}}{r}\right|_{2 \lambda_{1}, \Omega_{0 \varepsilon}}^{2}|v+\stackrel{\circ}{v}|_{2 \lambda_{2}, \Omega_{0 \varepsilon}}^{2}+\left|\frac{f_{r}}{r}\right|_{2, \Omega_{0 \varepsilon}}^{2}\right),
\end{aligned}
$$

where $1 / \lambda_{1}+1 / \lambda_{2}=1$. In view of the Poincaré inequality we can express (3.36) in the form

$$
\begin{aligned}
& \frac{d}{d t}\left|\frac{v_{r}}{r}\right|_{2, \Omega_{0 \varepsilon}}^{2}+\nu_{1}\left|\frac{v_{r}}{r}\right|_{2, \Omega_{0 \varepsilon}}^{2}+\nu_{2}\left(\left|\left(\frac{v_{r}}{r}\right)_{, r}\right|_{2, \Omega_{0 \varepsilon}}^{2}+\left|\left(\frac{v_{r}}{r}\right)_{, z}\right|_{2, \Omega_{0 \varepsilon}}^{2}\right) \\
& \leq c\left(\left|\frac{\chi}{r}\right|_{3, \Omega_{0 \varepsilon}}^{2}\left|v_{z}\right|_{2, \Omega_{0 \varepsilon}}^{2}+\left|\frac{p-\stackrel{\circ}{p}}{r}\right|_{2, \Omega_{0 \varepsilon}}^{2}+\left|\frac{v-\stackrel{\circ}{v}}{r}\right|_{2 \lambda_{1}, \Omega_{0 \varepsilon}}^{2}|v+\stackrel{\circ}{v}|_{2 \lambda_{2}, \Omega_{0 \varepsilon}}^{2}+\left|\frac{f_{r}}{r}\right|_{2, \Omega_{0 \varepsilon}}^{2}\right),
\end{aligned}
$$

where $1 / \lambda_{1}+1 / \lambda_{2}=1$. Multiplying (3.37) by $e^{\nu_{1} t}$ and integrating with respect to time from $k T$ to $t \in(k T,(k+1) T]$ yields

$$
\begin{aligned}
& \left|\frac{v_{r}(t)}{r}\right|_{2, \Omega_{0 \varepsilon}}^{2}+\nu_{2} e^{-\nu_{1} t} \int_{k T}^{t}\left(\left|\left(\frac{v_{r}\left(t^{\prime}\right)}{r}\right)_{, r}\right|_{2, \Omega_{0 \varepsilon}}^{2}+\left|\left(\frac{v_{r}\left(t^{\prime}\right)}{r}\right)_{, z}\right|_{2, \Omega_{0 \varepsilon}}^{2}\right) e^{\nu_{1} t^{\prime}} d t^{\prime} \\
& \leq c e^{-\nu_{1} t} \int_{k T}^{t}\left(\left|\frac{\chi}{r}\right|_{3, \Omega_{0 \varepsilon}}^{2}\left|v_{z}\right|_{2, \Omega_{0 \varepsilon}}^{2}+\left|\frac{p-\stackrel{\circ}{p}}{r}\right|_{2, \Omega_{0 \varepsilon}}^{2}\right. \\
& \left.+\left|\frac{v-\stackrel{\circ}{v}}{r}\right|_{2 \lambda_{1}, \Omega_{0 \varepsilon}}^{2}|v+\stackrel{\circ}{v}|_{2 \lambda_{1}, \Omega_{0 \varepsilon}}^{2}+\left|\frac{f_{r}}{r}\right|_{2, \Omega_{0 \varepsilon}}^{2}\right) e^{\nu_{1} t^{\prime}} d t^{\prime} \\
& +\left|\frac{v_{r}(k T)}{r}\right|_{2, \Omega_{0 \varepsilon}}^{2} e^{-\nu_{1}(t-k T)}
\end{aligned}
$$

where $1 / \lambda_{1}+1 / \lambda_{2}=1$. Now we shall estimate the first term on the r.h.s. of (3.38). In view of $(3.4)_{1}$ and $(3.22)$ we have that

$$
\begin{aligned}
& c e^{-\nu_{1} t} \int_{k T}^{t}\left|\frac{\chi}{r}\right|_{3, \Omega_{0 \varepsilon}}^{2}\left|v_{z}\right|_{2, \Omega_{0 \varepsilon}}^{2} e^{\nu_{1} t^{\prime}} d t^{\prime} \leq c e^{-\nu_{1} t} \int_{k T}^{t}\left|\frac{\chi}{r}\right|_{3, \Omega_{0}}^{2}\left|v_{z}\right|_{2, \Omega_{0}}^{2} e^{\nu_{1} t^{\prime}} d t^{\prime} \\
& \leq c A_{1}^{2}(T) A_{3}^{2}(3, T) e^{-\nu_{1} t} \int_{k T}^{t} e^{\nu_{1} t^{\prime}} d t^{\prime} \leq c A_{1}^{2}(T) A_{3}^{2}(3, T) .
\end{aligned}
$$

From (3.22) we have that

$$
|\chi(t)|_{s, \Omega_{0}} \leq R\left|\frac{\chi(t)}{r}\right|_{s, \Omega_{0}} \leq 2 R A_{3}(s, T) .
$$


Then considering the problem

$$
\begin{array}{ll}
\operatorname{rot} v=\alpha=\chi e_{\varphi} & \text { in } \Omega_{0 \varepsilon}, \\
\operatorname{div} v=0 & \text { in } \Omega_{0 \varepsilon}, \\
v \cdot \bar{n}=0 & \text { on } S_{0 \varepsilon},
\end{array}
$$

we obtain the estimate

$$
\|v\|_{1, s, \Omega_{0 \varepsilon}} \leq c|\chi|_{s, \Omega_{0 \varepsilon}} \leq c R A_{3}(s, T) .
$$

Let us consider the third expression under the time integral on the r.h.s. of (3.38). Let $\lambda_{1}=\frac{3}{2}, \lambda_{2}=3$. Then it is bounded by

$$
\left|\frac{v-\stackrel{\circ}{v}}{r}\right|_{3, \Omega_{0}}^{2}|v+\stackrel{\circ}{v}|_{6, \Omega_{0}}^{2} \equiv I_{1} .
$$

By the Hardy inequality the first factor in $I_{1}$ is estimated by

$$
c\left|v_{, r}\right|_{3, \Omega_{0}}^{2} \equiv I_{2},
$$

so in view of (3.41) we have

$$
I_{2} \leq c R^{2} A_{3}^{2}(3, T)
$$

In view of the imbedding

$$
\left.|v|_{r=0}\right|_{6, \Omega_{0}} \leq c\|v\|_{1,3, \Omega_{0}},
$$

the second factor in $I_{1}$ is bounded by

$$
c\|v\|_{1,3, \Omega_{0}}^{2} \leq c R^{2} A_{3}^{2}(3, T) .
$$

Hence,

$$
I_{1} \leq c R^{4} A_{3}^{4}(3, T) .
$$

Finally, the term with pressure under the time integral on the r.h.s. (3.38) is estimated by

$$
c\left|\frac{p-\stackrel{\circ}{p}}{r}\right|_{3, \Omega_{0 \varepsilon}} \leq c\left|\frac{p-\stackrel{\circ}{p}}{r}\right|_{3, \Omega_{0}} \leq c\left|p_{, r}\right|_{3, \Omega_{0}} \equiv I_{3},
$$

where in the second inequality the Hardy inequality was applied.

To estimate $I_{3}$ we consider the problem for pressure

$$
\begin{array}{ll}
\Delta p=-\nabla v \cdot \nabla v+\operatorname{div} f & \text { in } \Omega_{0}, \\
p_{, r}=f_{r} & \text { for } r=R, \\
p_{, z}=f_{z} & \text { for } z=\mp a .
\end{array}
$$

For solutions of problem (3.42) we have

$$
\|\nabla p\|_{1, \sigma, \Omega_{0}} \leq c\left(|\nabla v|_{2 \sigma, \Omega_{0}}^{2}+|\operatorname{div} f|_{\sigma, \Omega_{0}}+\|f\|_{1-1 / \sigma, \sigma, \partial \Omega_{0}}\right) .
$$

Hence

$$
\left|p_{, r}\right|_{3, \Omega_{0}} \leq\|\nabla p\|_{1,3 / 2, \Omega_{0}} \leq c\left(|\nabla v|_{3, \Omega_{0}}^{2}+|\operatorname{div} f|_{3, \Omega_{0}}+\|f\|_{1 / 3,3 / 2, \partial \Omega_{0}}\right) .
$$

Then (3.41) yields

$$
\left|p_{, r}\right|_{3, \Omega_{0}} \leq c\left(R^{2} A_{3}^{2}(3, T)+|\operatorname{div} f|_{3, \Omega_{0}}+\|f\|_{1 / 3,3 / 2, \partial \Omega_{0}}\right) .
$$


Summarizing, (3.38) takes the form

$$
\begin{aligned}
& \left|\frac{v_{r}(t)}{r}\right|_{2, \Omega_{0 \varepsilon}}^{2}+\nu_{2} e^{-\nu_{1} t} \int_{k T}^{t}\left(\left|\left(\frac{v_{r}\left(t^{\prime}\right)}{r}\right)_{, r}\right|_{2, \Omega_{0 \varepsilon}}^{2}+\left|\left(\frac{v_{r}\left(t^{\prime}\right)}{r}\right)_{, z}\right|_{2, \Omega_{0 \varepsilon}}^{2}\right) e^{\nu_{1} t^{\prime}} d t^{\prime} \\
& \leq c\left(A_{1}^{2}(T) A_{3}^{2}(3, T)+R^{4} A_{3}^{4}(3, T)\right) \\
& +c \int_{k T}^{t}\left(\left|\operatorname{div} f\left(t^{\prime}\right)\right|_{3, \Omega_{0}}^{2}+\left\|f\left(t^{\prime}\right)\right\|_{1 / 3,3 / 2, \partial \Omega_{0}}+\left|\frac{f_{r}\left(t^{\prime}\right)}{r}\right|_{2, \Omega_{0}}^{2}\right) d t^{\prime} \\
& +\left|\frac{v_{r}(k T)}{r}\right|_{2, \Omega_{0}}^{2} e^{-\nu_{1}(t-k T)} .
\end{aligned}
$$

In view of (3.31) inequality (3.46) takes the form

$$
\begin{aligned}
\left|\frac{v_{r}(t)}{r}\right|_{2, \Omega_{0 \varepsilon}}^{2}+\nu_{2} e^{-\nu_{1} t} \int_{k T}^{t}\left|\bar{\nabla} \frac{v_{r}}{r}\right|_{2, \Omega_{0 \varepsilon}}^{2} e^{\nu_{1} t^{\prime}} d t^{\prime} & \\
& \leq c B_{4}^{2}(T)+\left|\frac{v_{r}(k T)}{r}\right|_{2, \Omega_{0 \varepsilon}}^{2} e^{-\nu_{1}(t-k T)},
\end{aligned}
$$

where $t \in[k T,(k+1) T]$. Inserting $t=(k+1) T$ into (3.47) we obtain

$$
\left|\frac{v_{r}((k+1) T)}{r}\right|_{2, \Omega_{0 \varepsilon}}^{2} \leq c B_{4}^{2}(T)+\left|\frac{v_{r}(k T)}{r}\right|_{2, \Omega_{0 \varepsilon}}^{2} e^{-\nu_{1} T} .
$$

Hence by the inductive considerations we obtain

$$
\left|\frac{v_{r}(k T)}{r}\right|_{2, \Omega_{0 \varepsilon}}^{2} \leq \frac{c B_{4}^{2}(T)}{1-e^{-\nu_{1} T}}+e^{-\nu_{1} k T}\left|\frac{v_{r}(0)}{r}\right|_{2, \Omega_{0 \varepsilon}}^{2} .
$$

Passing with $\varepsilon$ to 0 yields (3.32).

Simplifying (3.47) yields

$$
\left|\frac{v_{r}(t)}{r}\right|_{2, \Omega_{0 \varepsilon}}^{2}+\nu_{2} e^{-\nu_{1} T} \int_{k T}^{t}\left|\bar{\nabla} \frac{v_{r}\left(t^{\prime}\right)}{r}\right|_{2, \Omega_{0 \varepsilon}}^{2} d t^{\prime} \leq c B_{4}^{2}(T)+\left|\frac{v_{r}(k T)}{r}\right|_{2, \Omega_{0 \varepsilon}}^{2},
$$

where $t \in[k T,(k+1) T]$. Passing with $\varepsilon$ to 0 and inserting $\nu_{1}=0, \nu_{2}=\nu$, we obtain from (3.50) inequality (3.33). This concludes the proof.

Finally we need

Lemma 3.5. Assume that $A_{5}(T)$ is defined by the r.h.s. of (3.60), $B_{6}(T)$ by (3.66), $A_{2}(T)$ by (3.12), $A_{4}(T)$ by (3.32). Then axially symmetric solutions to problem (1.1) are such that $v \in W_{2}^{2,1}\left(\Omega_{0} \times(k T,(k+1) T)\right) \cap C\left([k T,(k+1) T] ; H^{1}\left(\Omega_{0}\right)\right)$ for any $k \in \mathbb{N}$ and satisfy the inequalities

$$
\begin{aligned}
& \|\bar{v}\|_{2, \Omega_{0} \times(k T,(k+1) T)} \leq A_{6}(T), \\
& \|\bar{v}\|_{C\left([k T,(k+1) T] ; H^{1}\left(\Omega_{0}\right)\right)} \leq A_{7}(T),
\end{aligned}
$$

where 


$$
\begin{aligned}
& A_{6}(T)=c\left[A_{4}^{2}(T)+A_{5}^{2}(T)+B_{6}(T)+R A_{2}(T)\right], \\
& A_{7}(T)=c R A_{3}(2, T),
\end{aligned}
$$

where $A_{5}(T)$ is defined in (3.60) and $B_{6}(T)$ by (3.66).

Proof. To have the full norm of $H^{1}\left(\Omega_{0 \varepsilon}\right)$ in the second term on the l.h.s. of (3.5) we use the inequalitties

$$
\left|v_{r}\right|_{2, \Omega_{0 \varepsilon}}^{2} \leq R^{2}\left|\frac{v_{r}}{r}\right|_{2, \Omega_{0 \varepsilon}}^{2}
$$

and

$$
\left|v_{z}\right|_{2, \Omega_{0 \varepsilon}}^{2} \leq 4 a^{2}\left|v_{z, z}\right|_{2, \Omega_{0 \varepsilon}}^{2},
$$

where the second inequality is the Poincaré inequality. Then (3.5) assumes the form

$$
\frac{d}{d t}|\bar{v}|_{2, \Omega_{0 \varepsilon}}^{2}+\frac{\nu}{2} c_{3}\|\bar{v}\|_{1, \Omega_{0 \varepsilon}}^{2} \leq \frac{c_{1}}{\nu}|\bar{f}|_{6 / 5, \Omega_{0 \varepsilon}}^{2},
$$

where $c_{3}=\min \left\{1, \frac{1}{R^{2}}, \frac{1}{4 a^{2}}\right\}$. After the same considerations as in Lemma 3.1 we obtain

$$
\begin{aligned}
& |\bar{v}(k T)|_{2, \Omega_{0}}^{2} \leq A_{1}^{2}(T), \\
& |\bar{v}(t)|_{2, \Omega_{0}}^{2}+\frac{\nu}{2} c_{3} \int_{k T}^{t}\left\|\bar{v}\left(t^{\prime}\right)\right\|_{1, \Omega_{0}}^{2} d t^{\prime} \leq \frac{2}{\nu} A_{1}^{2}(T),
\end{aligned}
$$

for $t \in[k T,(k+1) T], k \in \mathbb{N}$.

From [3] we have

$$
\frac{1}{2} \frac{d}{d t}|\chi|_{2, \Omega_{0 \varepsilon}}^{2}+\nu\left(|\nabla \chi|_{2, \Omega_{0 \varepsilon}}^{2}+\left|\frac{\chi}{r}\right|_{2, \Omega_{0 \varepsilon}}^{2}\right) \leq c\left(\left\|v_{r}\right\|_{1, \Omega_{0 \varepsilon}}^{2}+|\bar{f}|_{2, \Omega_{0 \varepsilon}}^{2}\right) .
$$

Using that $\left.\chi\right|_{\partial \Omega_{0 \varepsilon}}=0$ we can express (3.54) in the form

$$
\frac{d}{d t}|\chi|_{2, \Omega_{0 \varepsilon}}^{2}+\nu\left(\|\chi\|_{1, \Omega_{0 \varepsilon}}^{2}+\left|\frac{\chi}{r}\right|_{2, \Omega_{0 \varepsilon}}^{2}\right) \leq c\left(\left\|v_{r}\right\|_{1, \Omega_{0 \varepsilon}}^{2}+|\bar{f}|_{2, \Omega_{0 \varepsilon}}^{2}\right) .
$$

Utilizing that $\nu=\nu_{1}+\nu_{2}$ and multiplying (3.55) by $e^{\nu_{1} t}$ we obtain

$$
\frac{d}{d t}\left(|\chi|_{2, \Omega_{0 \varepsilon}}^{2} e^{\nu_{1} t}\right)+\nu_{2}\left(\|\chi\|_{1, \Omega_{0 \varepsilon}}^{2}+\left|\frac{\chi}{r}\right|_{2, \Omega_{0 \varepsilon}}^{2}\right) e^{\nu_{1} t} \leq c\left(\left\|v_{r}\right\|_{1, \Omega_{0 \varepsilon}}^{2}+|\bar{f}|_{2, \Omega_{0 \varepsilon}}^{2}\right) e^{\nu_{1} t} .
$$

Integrating (3.56) with respect to time from $k T$ to $t \in(k T,(k+1) T]$ and neglecting the second term on the l.h.s. we obtain

$$
|\chi(t)|_{2, \Omega_{0 \varepsilon}}^{2} \leq c e^{-\nu_{1} t} \int_{k T}^{t}\left(\left\|v_{r}\left(t^{\prime}\right)\right\|_{1, \Omega_{0 \varepsilon}}^{2}+\left|\bar{f}\left(t^{\prime}\right)\right|_{2, \Omega_{0 \varepsilon}}^{2}\right) e^{\nu_{1} t^{\prime}} d t^{\prime}+|\chi(k T)|_{2, \Omega_{0 \varepsilon}}^{2} e^{-\nu_{1}(t-k T)}
$$

Inserting $t=(k+1) T$ and utilizing (3.53) we get

$$
|\chi((k+1) T)|_{2, \Omega_{0 \varepsilon}}^{2} \leq c B_{5}^{2}(T)+|\chi(k T)|_{2, \Omega_{0 \varepsilon}}^{2} e^{-\nu_{1} T},
$$

where

$$
B_{5}^{2}(T)=A_{1}^{2}(T)+\sup _{k \in \mathbb{N}} \int_{k T}^{(k+1) T}\left|\bar{f}\left(t^{\prime}\right)\right|_{2, \Omega_{0}}^{2} d t^{\prime} .
$$


Inductive considerations and passing with $\varepsilon$ to 0 implies

(3.60) $|\chi(k T)|_{2, \Omega_{0}}^{2} \leq \frac{c B_{5}^{2}(T)}{1-e^{-\nu_{1} T}}+|\chi(0)|_{2, \Omega_{0}}^{2} e^{-\nu_{1} k T} \leq \frac{c B_{5}^{2}(T)}{1-e^{-\nu_{1} T}}+|\chi(0)|_{2, \Omega_{0}}^{2} \equiv A_{5}^{2}(T)$.

Integrating (3.55) with respect to time from $k T$ to $t \in(k T,(k+1) T]$, passing with $\varepsilon$ to 0 , utilizing $(3.53)_{2}$ and $(3.60)$ we have

$$
|\chi(t)|_{2, \Omega_{0}}^{2}+\nu \int_{k T}^{t}\left(\left\|\chi\left(t^{\prime}\right)\right\|_{1, \Omega_{0}}^{2}+\left|\frac{\chi\left(t^{\prime}\right)}{r}\right|_{2, \Omega_{0}}^{2}\right) d t^{\prime} \leq c A_{5}^{2}(T) .
$$

Let us consider the problem

$$
\begin{array}{ll}
v_{r, z}-v_{z, r}=\chi & \text { in } \Omega_{0 \varepsilon}, \\
v_{r, z}+v_{z, z}=-\frac{v_{r}}{r} & \text { in } \Omega_{0 \varepsilon}, \\
\left.v_{r}\right|_{r=R, \varepsilon}=0,\left.\quad v_{z}\right|_{z=\mp a}=0 . &
\end{array}
$$

In view of (3.33) and (3.61) we have

$$
\begin{aligned}
& \|\bar{v}\|_{L_{\infty}\left(k T, t ; H^{1}\left(\Omega_{0 \varepsilon}\right)\right)}+\|\bar{v}\|_{L_{2}\left(k T, t ; H^{2}\left(\Omega_{0 \varepsilon}\right)\right)} \\
& \leq c\left(A_{4}(T)+A_{5}(T)\right), \quad t \in(k T,(k+1) T], \quad k \in \mathbb{N} .
\end{aligned}
$$

Passing with $\varepsilon$ to 0 yields

$$
\begin{aligned}
& \|\bar{v}\|_{L_{\infty}\left(k T, t ; H^{1}\left(\Omega_{0}\right)\right)}+\|\bar{v}\|_{L_{2}\left(k T, t ; H^{2}\left(\Omega_{0}\right)\right)} \\
& \leq c\left(A_{4}(T)+A_{5}(T)\right), \quad t \in(k T,(k+1) T], \quad k \in \mathbb{N} .
\end{aligned}
$$

Let us consider the problem

$$
\begin{array}{ll}
\bar{v}_{, t}-\operatorname{div} \mathbb{T}(\bar{v}, p)=-\bar{v} \cdot \nabla \bar{v}+f & \text { in } \Omega_{0} \times(k T,(k+1) T), \\
\operatorname{div} \bar{v}=0 & \text { in } \Omega_{0} \times(k T,(k+1) T), \\
\bar{v} \cdot \bar{n}=0, \quad \bar{n} \cdot \mathbb{T}(\bar{v}, p) \cdot \bar{\tau}_{\alpha}=0, \quad \alpha=1,2, & \text { on } S_{0} \times(k T,(k+1) T), \\
\left.\bar{v}\right|_{t=k T}=\bar{v}(k T) & \text { in } \Omega_{0} .
\end{array}
$$

In view of (3.64) we have

$$
\begin{gathered}
|\bar{v} \cdot \nabla \bar{v}|_{2, \Omega_{0} \times(k T,(k+1) T)} \leq\|\bar{v}\|_{L_{\infty}\left(k T,(k+1) T ; L_{4}\left(\Omega_{0}\right)\right)} \cdot \\
\cdot\|\nabla \bar{v}\|_{L_{2}\left(k T,(k+1) T ; L_{4}\left(\Omega_{0}\right)\right)} \leq c\left(A_{4}^{2}(T)+A_{5}^{2}(T)\right) .
\end{gathered}
$$

Assuming that

$$
B_{6}(T)=\sup _{k \in \mathbb{N}}|\bar{f}|_{2, \Omega_{0} \times(k T,(k+1) T)}
$$

we obtain for solutions of problem (3.65) the estimate

$$
\|\bar{v}\|_{2, \Omega_{0} \times(k T,(k+1) T)} \leq c\left(A_{4}^{2}(T)+A_{5}^{2}(T)+B_{6}(T)+\|\bar{v}(k T)\|_{1, \Omega_{0}}\right) .
$$

Finally, we have to show that $\|\bar{v}(k T)\|_{1, \Omega_{0}}$ can be estimated by a constant independent of $k$. For this purpose we use problem (3.40). Since (3.12) holds we have

$$
\|\bar{v}(k T)\|_{1, \Omega_{0}} \leq c R\left|\frac{\chi(k T)}{r}\right|_{2, \Omega_{0}} \leq c R A_{2}(T) .
$$


From (3.67) and (3.68) estimate $(3.51)_{1}$ follows. Finally to show $(3.51)_{2}$ we recall that

$$
\frac{d}{d t}\left|\frac{\chi}{r}\right|_{s, \Omega_{0 \varepsilon}} \leq\left|\frac{F}{r}\right|_{s, \Omega_{0 \varepsilon}},
$$

so $\left|\frac{\chi}{r}\right|_{s, \Omega_{0}} \in C\left(\mathbb{R}_{+}\right)$. Hence by (3.40) we obtain that $\bar{v} \in C\left(\mathbb{R}_{+} ; W_{s}^{1}\left(\Omega_{0}\right)\right)$, so $(3.51)_{2}$ holds. This concludes the proof.

Finally, we derive an explicit form of estimate (3.5).

REMARK 3.6. First we have to estimate expressions from the r.h.s. of (3.5). Let $T$ be so large that $e^{-\nu_{1} T / 2} \leq \frac{1}{2}$. From Lemma 3.1 we have

$$
A_{1}(T) \leq c\left(\sup _{k}|f|_{6 / 5,2, \Omega_{0} \times(k T,(k+1) T)}+|\bar{v}(0)|_{2, \Omega_{0}}\right) .
$$

Lemma 3.2 implies

$$
A_{2}(T) \leq c\left(\sup _{k}\left|\frac{f}{r}\right|_{2, \Omega_{0} \times(k T,(k+1) T)}+\left|\frac{\chi(0)}{r}\right|_{2, \Omega_{0}}\right) .
$$

Lemma 3.3 yields

$$
A_{3}(3, T) \leq c\left(\sup _{k}\left|\frac{F}{r}\right|_{3, \Omega_{0} \times(k T,(k+1) T)}+\left|\frac{\chi(0)}{r}\right|_{3, \Omega_{0}}\right) .
$$

Lemma 3.4 shows that

$$
\begin{aligned}
A_{4}(T) \leq & c\left[A_{1}(T) A_{3}(3, T)+R^{2} A_{3}^{2}(3, T)\right. \\
& +\sup _{k}\left(|\operatorname{div} f|_{3,2, \Omega_{0} \times(k T,(k+1) T)}+\|f\|_{L_{2}\left(k T,(k+1) T ; W_{3 / 2}^{1 / 3}\left(\partial \Omega_{0}\right)\right)}\right. \\
& \left.\left.+\left|\frac{f_{r}}{r}\right|_{2, \Omega_{0} \times(k T,(k+1) T)}\right)\right]+\left|\frac{v_{r}(0)}{r}\right|_{2, \Omega_{0}} .
\end{aligned}
$$

From (3.59) and (3.60) we have

$$
A_{5}(T) \leq c\left(\sup _{k}|f|_{2, \Omega_{0} \times(k T,(k+1) T)}+|v(0)|_{2, \Omega_{0}}+|\chi(0)|_{2, \Omega_{0}}\right) .
$$

Finally,

$$
B_{6}(T)=\sup _{k \in \mathbb{N}}|\bar{f}|_{2, \Omega_{0} \times(k T,(k+1) T)} .
$$

In view of the above estimates, (3.51) takes the form

$$
\begin{aligned}
& \|\bar{v}\|_{2, \Omega_{0} \times(k T,(k+1) T)} \leq c \sup _{k \in \mathbb{N}}\left(|f|_{2, \Omega_{0} \times(k T,(k+1) T)}^{4}+\left|\frac{f_{r}}{r}\right|_{2, \Omega_{0} \times(k T,(k+1) T)}\right. \\
& +\left|\frac{f_{r}}{r}\right|_{2, \Omega_{0} \times(k T,(k+1) T)}^{2}+|\operatorname{div} f|_{3,2, \Omega_{0} \times(k T,(k+1) T)}^{2} \\
& \left.+\left|\frac{F}{r}\right|_{3, \Omega_{0} \times(k T,(k+1) T)}^{4}\right) \\
& \left.+\|f\|_{L_{2}\left(k T,(k+1) T ; W_{3 / 2}^{1 / 3}\left(\partial \Omega_{0}\right)\right)}^{2}\right)
\end{aligned}
$$




$$
+c\left(|\bar{v}(0)|_{2, \Omega_{0}}^{4}+|\bar{v}(0)|_{2, \Omega_{0}}^{2}+\left|\frac{\chi(0)}{r}\right|_{3, \Omega_{0}}^{4}+\left|\frac{\chi(0)}{r}\right|_{2, \Omega_{0}}+|\chi(0)|_{2, \Omega_{0}}^{2}\right)
$$

and

$$
\|v\|_{C\left([k T,(k+1) T] ; H^{1}\left(\Omega_{0}\right)\right)} \leq c\left(\sup _{k}\left|\frac{F}{r}\right|_{2, \Omega_{0} \times(k T,(k+1) T)}+\left|\frac{\chi(0)}{r}\right|_{2, \Omega_{0}}\right) .
$$

From Lemmas 3.1-3.5, Remark 3.6 and the result of global existence of regular axially symmetric solutions proved in [3] we obtain Theorem 1.

4. Stability problem. First we obtain an $L_{2}$-estimate for $v^{\prime}$.

LEMMA 4.1. Assume that $v_{a} \in L_{\infty}\left(k T,(k+1) T ; W_{3}^{1}\left(\Omega_{0}\right)\right), \frac{f_{a}}{r} \in L_{2}\left(k T(k+1) T ; L_{2}(\Omega)\right)$, $k \in \mathbb{N}, \frac{\chi(0)}{r} \in L_{2}\left(\Omega_{0}\right)$. Let $\nu_{1}^{\prime}<\nu^{\prime}=\frac{\nu}{c_{1}}$ and $c_{1}$ is from (4.3). Let $T$ be so large that

$$
\alpha(T) \equiv \frac{1}{\nu^{2}} \sup _{k}\left|\frac{f_{a}}{r}\right|_{2, \Omega \times(k T,(k+1) T)}^{2}+\frac{1}{\nu}\left|\frac{\chi(k T)}{r}\right|_{2, \Omega}^{2} \leq \frac{\nu_{1}^{\prime}}{2} T .
$$

Then

$$
\left|v^{\prime}((k+1) T)\right|_{2, \Omega}^{2} \leq D_{1}^{2}(T)+e^{-\left(\nu_{1}^{\prime} / 2\right) T}\left|v^{\prime}(k T)\right|_{2, \Omega}^{2},
$$

where

$$
D_{1}^{2}(T)=\sup _{k \in \mathbb{N}} c_{3} e^{\alpha(T)} \int_{k T}^{(k+1) T}\left(\left|f^{\prime}(t)\right|_{2, \Omega}^{2}+\left|\int_{\Omega} r v_{\varphi}^{\prime}(t) d x\right|^{2}\right) d t .
$$

Proof. Multiplying (1.9) by $v^{\prime}$, integrating over $\Omega$ and using the boundary conditions we obtain

$$
\frac{1}{2} \frac{d}{d t}\left|v^{\prime}\right|_{2, \Omega}^{2}+\nu\left|\mathbb{D}\left(v^{\prime}\right)\right|_{2, \Omega}^{2}+\int_{\Omega} v^{\prime} \cdot \nabla v_{a} \cdot v^{\prime} d x=\int_{\Omega} f^{\prime} \cdot v^{\prime} d x .
$$

Utilizing the Korn inequality (see [8, Ch. 4])

$$
\left\|v^{\prime}(t)\right\|_{1, \Omega}^{2} \leq c_{1}\left(\left|\mathbb{D}\left(v^{\prime}\right)\right|_{2, \Omega}^{2}+\left|\int_{\Omega} r v_{\varphi}^{\prime}(t) d x\right|^{2}\right)
$$

in $(4.2)$ yields

$$
\frac{1}{2} \frac{d}{d t}\left|v^{\prime}\right|_{2, \Omega}^{2}+\nu^{\prime}\left\|v^{\prime}\right\|_{1, \Omega}^{2}+\int_{\Omega} v^{\prime} \cdot \nabla v_{a} \cdot v^{\prime} d x \leq \int_{\Omega} f^{\prime} \cdot v^{\prime} d x+\left|\int_{\Omega} r v_{\varphi}^{\prime}(t) d x\right|^{2},
$$

where $\nu^{\prime}=\nu / c_{1}$. Applying the Hölder and Young inequalities in (4.4) implies

$$
\begin{aligned}
& \frac{d}{d t}\left|v^{\prime}\right|_{2, \Omega}^{2}+\nu_{1}^{\prime}\left|v^{\prime}\right|_{2, \Omega}^{2}+\nu_{2}^{\prime}\left\|v^{\prime}\right\|_{1, \Omega}^{2} \leq c_{2}\left|\nabla v_{a}\right|_{3, \Omega}^{2}\left|v^{\prime}\right|_{2, \Omega}^{2} \\
& +c\left|f^{\prime}\right|_{6 / 5, \Omega}^{2}+\left|\int_{\Omega} r v_{\varphi}^{\prime}(t) d x\right|^{2} .
\end{aligned}
$$

Continuing, we have

$$
\frac{d}{d t}\left(\left|v^{\prime}\right|_{2, \Omega}^{2} e^{\nu_{1}^{\prime} t-c_{2} \int_{k T}^{t}\left|\nabla v_{a}\right|_{3, \Omega}^{2} d t^{\prime}}\right)+\nu_{2}^{\prime}\left\|v^{\prime}\right\|_{1, \Omega}^{2} e^{\nu_{1}^{\prime} t-c_{2} \int_{k T}^{t}\left|\nabla v_{a}\right|_{3, \Omega}^{2} d t^{\prime}}
$$




$$
\leq c_{3}\left(\left|f^{\prime}\right|_{6 / 5, \Omega}^{2}+\left|\int_{\Omega} r v_{\varphi}^{\prime}(t) d x\right|^{2}\right) e^{\nu_{1}^{\prime} t-c_{2} \int_{k T}^{t}\left|\nabla v_{a}\right|_{3, \Omega}^{2} d t^{\prime}} .
$$

Integrating (4.6) with respect to $t$ from $k T$ to $t \in[k T,(k+1) T]$ we obtain

$$
\begin{aligned}
& \left|v^{\prime}(t)\right|_{2, \Omega}^{2} e^{\nu_{1}^{\prime} t-c_{2} \int_{k T}^{t}\left|\nabla v_{a}\left(t^{\prime}\right)\right|_{3, \Omega}^{2} d t^{\prime}}+\nu_{2}^{\prime} \int_{k T}^{t}\left\|v^{\prime}\left(t^{\prime}\right)\right\|_{1, \Omega}^{2} e^{\nu_{1}^{\prime} t^{\prime}-c_{2} \int_{k T}^{t^{\prime}}\left|\nabla v_{a}\left(t^{\prime \prime}\right)\right|_{3, \Omega}^{2} d t^{\prime \prime}} d t^{\prime} \\
& \leq c_{3} \int_{k T}^{t}\left(\left|f^{\prime}\left(t^{\prime}\right)\right|_{6 / 5, \Omega}^{2}+\left|\int_{\Omega} r v_{\varphi}^{\prime}\left(t^{\prime}\right) d x\right|^{2}\right) e^{\nu_{1}^{\prime} t^{\prime}-c_{2} \int_{k T}^{t^{\prime}}\left|\nabla v_{a}\left(t^{\prime \prime}\right)\right|_{3, \Omega}^{2} d t^{\prime \prime}} d t^{\prime} \\
& +\left|v^{\prime}(k T)\right|_{2, \Omega}^{2} e^{\nu_{1}^{\prime} k T}
\end{aligned}
$$

Simplifying (4.7) yields

$$
\begin{aligned}
& \left|v^{\prime}(t)\right|_{2, \Omega}^{2}+\nu_{2}^{\prime} e^{-\nu_{1}^{\prime} t} \int_{k T}^{t}\left\|v^{\prime}\left(t^{\prime}\right)\right\|_{1, \Omega}^{2} e^{\nu_{1}^{\prime} t^{\prime}} d t^{\prime} \\
& \leq c_{3} e^{c_{2} \int_{k T}^{t}\left|\nabla v_{a}\right|_{3, \Omega}^{2} d t^{\prime}} \int_{k T}^{t}\left(\left|f^{\prime}\left(t^{\prime}\right)\right|_{6 / 5, \Omega}^{2}+\left|\int_{\Omega} r v_{\varphi}^{\prime}\left(t^{\prime}\right) d x\right|^{2}\right) d t^{\prime} \\
& +e^{-\nu_{1}^{\prime}(t-k T)+c_{2} \int_{k T}^{t}\left|\nabla v_{a}\left(t^{\prime}\right)\right|_{3, \Omega}^{2} d t^{\prime}}\left|v^{\prime}(k T)\right|_{2, \Omega}^{2},
\end{aligned}
$$

where $t \in[k T,(k+1) T]$. Inserting $t=(k+1) T$ and cancelling the second term on the 1.h.s. of (4.8) implies

$$
\begin{aligned}
& \left|v^{\prime}((k+1) T)\right|_{2, \Omega}^{2} \leq c_{3} e^{c_{2} \int_{k T}^{(k+1) T}\left|\nabla v_{a}\left(t^{\prime}\right)\right|_{3, \Omega}^{2} d t^{\prime}} \int_{k T}^{(k+1) T}\left(\left.f^{\prime}(t)\right|_{2, \Omega} ^{2}+\left|\int_{\Omega} r v_{\varphi}(t) d x\right|^{2}\right) d t \\
& +e^{-\nu_{1}^{\prime} T+c_{2} \int_{k T}^{(k+1) T}}\left|\nabla v_{a}(t)\right|_{3, \Omega}^{2} d t\left|v^{\prime}(k T)\right|_{2, \Omega}^{2} .
\end{aligned}
$$

In view of (3.8) we have

$$
\int_{k T}^{(k+1) T}\left|\nabla v_{a}(t)\right|_{3, \Omega}^{2} d t \leq \frac{1}{\nu^{2}}\left|\frac{f_{a}}{r}\right|_{2, \Omega \times(k T,(k+1) T)}^{2}+\frac{1}{\nu}\left|\frac{\chi(k T)}{r}\right|_{2, \Omega}^{2} \leq \alpha(T) .
$$

Utilizing the inequality and assumptions of the lemma we obtain (4.1). This ends the proof.

By the inductive considerations we obtain from (4.1) the estimate

$$
\left|v^{\prime}(k T)\right|_{2, \Omega}^{2} \leq \frac{D_{1}^{2}(T)}{1-e^{-\left(\nu_{1}^{\prime} / 2\right) T}}+e^{-\frac{\nu^{\prime}}{2} k T}\left|v^{\prime}(0)\right|_{2, \Omega}^{2} \leq \frac{D_{1}^{2}(T)}{1-e^{-\left(\nu_{1}^{\prime} / 2\right) T}}+\left|v^{\prime}(0)\right|_{2, \Omega}^{2} \equiv G_{1}(T) .
$$

Moreover, from (4.8) we have

$$
\left|v^{\prime}(t)\right|_{2, \Omega}^{2}+\nu_{2}^{\prime} e^{-\nu_{1}^{\prime} t} \int_{k T}^{t}\left\|v^{\prime}\left(t^{\prime}\right)\right\|_{1, \Omega}^{2} e^{\nu_{1}^{\prime} t^{\prime}} d t^{\prime} \leq D_{1}^{2}(T)+e^{-\left(\nu_{1}^{\prime} / 2\right) T}\left|v^{\prime}(k T)\right|_{2, \Omega}^{2},
$$


where $t \in[k T,(k+1) T]$. From (4.11) we obtain the estimates

$$
\begin{aligned}
& \left|v^{\prime}(t)\right|_{2, \Omega}^{2} \leq D_{1}^{2}(T)+\left|v^{\prime}(k T)\right|_{2, \Omega}^{2}, \\
& \int_{k T}^{t}\left\|v^{\prime}\left(t^{\prime}\right)\right\|_{1, \Omega}^{2} d t^{\prime} \leq e^{\nu_{1}^{\prime} T}\left(D_{1}^{2}(T)+\left|v^{\prime}(k T)\right|_{2, \Omega}^{2}\right),
\end{aligned}
$$

where $t \in[k T,(k+1) T]$.

Now we prove the existence of local solutions to problem (1.9).

Lemma 4.2. Let the assumptions of Theorem 1 be satisfied. Let $v^{\prime}(0) \in L_{2}(\Omega), f^{\prime} \in$ $L_{2}\left(\Omega \times(k T,(k+1) T)\right.$ for any $k \in \mathbb{N}$. Let $D_{1}(T)$ (see $\left.\left(4.1^{\prime}\right)\right), G_{1}(T)$ (see (4.10)), $D_{2}(T)$ (see (4.14)) be sufficiently small. Let

$$
\beta(k)=D_{2}(T)\left(A_{6}^{6}(T)+A_{6}^{4}(T)\right)+\left(\left|f^{\prime}\right|_{2, \Omega \times(k T,(k+1) T)}+\left\|v^{\prime}(k T)\right\|_{1, \Omega}\right)
$$

and let there exist a number $\sigma>1$ such that

$$
c D_{2} \sigma^{5} \beta^{5}+\frac{c}{\sigma} \leq 1
$$

Then there exists a solution to problem (1.9) such that $v^{\prime} \in W_{2}^{2,1}(\Omega \times(k T,(k+1) T))$ and

$$
\left\|v^{\prime}\right\|_{2, \Omega \times(k T,(k+1) T)}+\left|\nabla p^{\prime}\right|_{2, \Omega \times(k T,(k+1) T)} \leq A(k),
$$

where $A(k)$ defined by inequality (4.19) is such that $A(k) \leq \sigma \beta(k)$.

Proof. To prove the existence of solutions to problem (1.9) we apply the following method of successive approximations:

$$
\begin{aligned}
& v_{m+1, t}^{\prime}+v_{m}^{\prime} \cdot \nabla v_{m+1}^{\prime}+v_{m+1}^{\prime} \cdot \nabla v_{a}+v_{a} \cdot \nabla v_{m+1}^{\prime}-\operatorname{div} \mathbb{T}\left(v_{m+1}^{\prime}, p_{m+1}^{\prime}\right)=f^{\prime}, \\
& \operatorname{div} v_{m+1}^{\prime}=0, \\
& \bar{n} \cdot v_{m+1}^{\prime}=0, \\
& \bar{n} \cdot \mathbb{T}\left(v_{m+1}^{\prime}, p_{m+1}^{\prime}\right) \cdot \bar{\tau}_{\alpha}=0, \quad \alpha=1,2, \\
& \left.v_{m+1}^{\prime}\right|_{t=k T}=v^{\prime}(k T) .
\end{aligned}
$$

By (4.11) the weak solution to problem (4.13) satisfies the inequality

$$
\left|v_{m+1}^{\prime}(t)\right|_{2, \Omega}^{2}+\nu \int_{k T}^{t}\left\|v_{m+1}^{\prime}\left(t^{\prime}\right)\right\|_{1, \Omega}^{2} d t^{\prime} \leq\left(1+e^{\nu_{1}^{\prime} T}\right)\left[D_{1}^{2}(T)+\left|v^{\prime}(k T)\right|_{2, \Omega}^{2}\right] \equiv D_{2}^{2}(T)
$$

Let $c_{4}$ be the constant in the estimate of the Stokes system for (4.13). Then for solutions of (4.13) we have

$$
\begin{aligned}
& \left\|v_{m+1}^{\prime}\right\|_{2, \Omega \times(k T,(k+1) T)}+\left|\nabla p_{m+1}^{\prime}\right|_{2, \Omega \times(k T,(k+1) T)} \\
& \leq c_{4}\left(\left|v_{m}^{\prime} \cdot \nabla v_{m+1}^{\prime}\right|_{2, \Omega \times(k T,(k+1) T)}\right. \\
& +\left|v_{m+1}^{\prime} \cdot \nabla v_{a}\right|_{2, \Omega \times(k T,(k+1) T)}+\left|v_{a} \cdot \nabla v_{m+1}^{\prime}\right|_{2, \Omega \times(k T,(k+1) T)} \\
& \left.+\left|f^{\prime}\right|_{2, \Omega \times(k T,(k+1) T)}+\left\|v_{m+1}^{\prime}(k T)\right\|_{1, \Omega}\right) .
\end{aligned}
$$

Now, we estimate the particular terms on the r.h.s. of (4.15). We estimate the first and 
the third terms by

$$
\begin{aligned}
& \left|v_{m}^{\prime}+v_{a}\right|_{6, \Omega \times(k T,(k+1) T)}\left|\nabla v_{m+1}^{\prime}\right|_{3, \Omega \times(k T,(k+1) T)} \\
& \leq\left(\varepsilon^{1 / 6}\left\|\nabla v_{m+1}^{\prime}\right\|_{1, \Omega \times(k T,(k+1) T)}+c \varepsilon^{-5 / 6}\left|\nabla v_{m+1}^{\prime}\right|_{2, \Omega \times(k T,(k+1) T)}\right) . \\
& \cdot\left|v_{m}^{\prime}+v_{a}\right|_{6, \Omega \times(k T,(k+1) T)} \\
& \leq \varepsilon_{1}^{1 / 6}\left\|\nabla v_{m+1}^{\prime}\right\|_{1, \Omega \times(k T,(k+1) T)}+c\left(1 / \varepsilon_{1}\right)\left|v_{m}^{\prime}+v_{a}\right|_{6, \Omega \times(k T,(k+1) T)}^{6} D_{2}(T) \\
& \leq \varepsilon_{1}^{1 / 6}\left\|\nabla v_{m+1}^{\prime}\right\|_{1, \Omega \times(k T,(k+1) T)}+c\left(1 / \varepsilon_{1}\right) D_{2}(T) . \\
& \cdot\left(\left\|v_{m}^{\prime}\right\|_{2, \Omega \times(k T,(k+1) T)}^{6}+\left\|v_{a}\right\|_{2, \Omega \times(k T,(k+1) T)}^{6}\right) .
\end{aligned}
$$

By the Hölder inequality the second term on the r.h.s. of (4.15) is bounded by

$$
\begin{aligned}
& \left|v_{m+1}^{\prime}\right|_{5, \Omega \times(k T,(k+1) T)}\left|\nabla v_{a}\right|_{\frac{10}{3}, \Omega \times(k T,(k+1) T)} \leq\left[\varepsilon_{2}^{1 / 4}\left\|v_{m+1}^{\prime}\right\|_{2, \Omega \times(k T,(k+1) T)}\right. \\
& \left.\quad+c \varepsilon_{2}^{-3 / 4}\left|v_{m+1}^{\prime}\right|_{10 / 3, \Omega \times(k T,(k+1) T)}\right] A_{6}(T) \\
& \leq \varepsilon_{3}^{1 / 4}\left\|v_{m+1}^{\prime}\right\|_{2, \Omega \times(k T,(k+1) T)}+c \varepsilon_{2}^{-3 / 4} c(T) D_{2}(T) A_{6}^{4}(T) .
\end{aligned}
$$

Utilizing the above estimates in (4.15) yields

$$
\begin{aligned}
& \left\|v_{m+1}^{\prime}\right\|_{2, \Omega \times(k T,(k+1) T)}+\left|\nabla p_{m+1}^{\prime}\right|_{2, \Omega \times(k T,(k+1) T)} \\
& \leq c D_{2}(T)\left(\left\|v_{m}^{\prime}\right\|_{2, \Omega \times(k T,(k+1) T)}^{6}\right. \\
& \left.+A_{6}^{6}(T)+A_{6}^{4}(T)\right)+c_{4}\left(\left|f^{\prime}\right|_{2, \Omega \times(k T,(k+1) T)}+\left\|v^{\prime}(k T)\right\|_{1, \Omega}\right),
\end{aligned}
$$

where by (4.14) and (4.10) we have

$$
D_{2}(T)=\left(1+e^{\nu_{1}^{\prime} T}\right)\left[\frac{2-e^{-\left(\nu_{1}^{\prime} / 2\right) T}}{1-e^{-\left(\nu_{1}^{\prime} / 2\right) T}} D_{1}(T)+\left|v^{\prime}(0)\right|_{2, \Omega}\right] .
$$

To show that the constructed sequence $\left\{v_{m}^{\prime}, p_{m}^{\prime}\right\}$ is uniformly bounded we assume that

$$
\left\|v_{m}^{\prime}\right\|_{2, \Omega \times(k T,(k+1) T)}+\left|\nabla p_{m}^{\prime}\right|_{2, \Omega \times(k T,(k+1) T)} \leq A(k) .
$$

Next for $D_{2}(T)$ and $A(k)$ sufficiently small we get

$$
\begin{aligned}
& c D_{2}(T) A^{6}(k)+c D_{2}(T)\left(A_{6}^{6}(T)+A_{6}^{4}(T)\right)+c_{4}\left(\left|f^{\prime}\right|_{2, \Omega \times(k T,(k+1) T)}+\left\|v^{\prime}(k T)\right\|_{1, \Omega}\right) \\
& \leq A(k) .
\end{aligned}
$$

Then (4.19) implies

$$
\left\|v_{m+1}^{\prime}\right\|_{2, \Omega \times(k T,(k+1) T)}+\left|\nabla p_{m+1}^{\prime}\right|_{2, \Omega \times(k T,(k+1) T)} \leq A(k) .
$$

To show (4.18) for all $m \in \mathbb{N}$ we assume that $v_{0}^{\prime}=0$ and $v_{1}^{\prime}$ is a solution to the problem

$$
\begin{aligned}
& v_{1, t}^{\prime}+v_{1}^{\prime} \cdot \nabla v_{a}+v_{a} \cdot \nabla v_{1}^{\prime}-\operatorname{div} \mathbb{T}\left(v_{1}^{\prime}, p_{1}^{\prime}\right)=f^{\prime}, \\
& \operatorname{div} v_{1}^{\prime}=0, \\
& \bar{n} \cdot v_{1}^{\prime}=0, \quad \bar{n} \cdot \mathbb{T}\left(v_{1}^{\prime}, p_{1}^{\prime}\right) \cdot \bar{\tau}_{\alpha}=0, \quad \alpha=1,2, \\
& \left.v_{1}^{\prime}\right|_{t=k T}=v^{\prime}(k T) .
\end{aligned}
$$

For solutions of problem (4.21) we have

$$
\begin{aligned}
& \left\|v_{1}^{\prime}\right\|_{2, \Omega \times(k T,(k+1) T)} \leq c_{4}\left[\left|v_{1}^{\prime} \cdot \nabla v_{a}\right|_{2, \Omega \times(k T,(k+1) T)}\right. \\
& \left.+\left|v_{a} \cdot \nabla v_{1}^{\prime}\right|_{2, \Omega \times(k T,(k+1) T)}+\left|f^{\prime}\right|_{2, \Omega \times(k T,(k+1) T)}+\left\|v^{\prime}(k T)\right\|_{1, \Omega}\right] .
\end{aligned}
$$


Repeating the considerations leading to (4.16) we obtain

$$
\begin{aligned}
& \left\|v_{1}^{\prime}\right\|_{2, \Omega \times(k T,(k+1) T)}+\left|\nabla p_{1}^{\prime}\right|_{2, \Omega \times(k T,(k+1) T)} \leq c D_{2}(T)\left(A_{6}^{6}(T)+A_{6}^{4}(T)\right) \\
& +c_{4}\left(\left|f^{\prime}\right|_{2, \Omega \times(k T,(k+1) T)}+\left\|v^{\prime}(k T)\right\|_{1, \Omega}\right) .
\end{aligned}
$$

Assuming that

$$
c D_{2}(T)\left(A_{6}^{6}(T)+A_{6}^{4}(T)\right)+c_{4}\left(\left|f^{\prime}\right|_{2, \Omega \times(k T,(k+1) T)}+\left\|v^{\prime}(k T)\right\|_{1, \Omega}\right) \leq A(k),
$$

which is also justified by (4.19) we see by the inductive argument that (4.18) holds for any $m \in \mathbb{N}$.

Finally we must show convergence. Let $V_{m+1}^{\prime}=v_{m+1}^{\prime}-v_{m}^{\prime}, P_{m+1}^{\prime}=p_{m+1}^{\prime}-p_{m}^{\prime}$. Then (4.19) implies

$$
\begin{aligned}
& V_{m+1, t}^{\prime}+v_{m}^{\prime} \cdot \nabla V_{m+1}^{\prime}+V_{m}^{\prime} \cdot \nabla v_{m}^{\prime}+V_{m+1}^{\prime} \cdot \nabla v_{a}+v_{a} \cdot \nabla V_{m+1}^{\prime} \\
& -\operatorname{div} \mathbb{T}\left(V_{m+1}^{\prime}, P_{m+1}^{\prime}\right)=0, \\
& \operatorname{div} V_{m+1}^{\prime}=0, \\
& \bar{n} \cdot V_{m+1}^{\prime}=0, \quad \bar{n} \cdot \mathbb{T}\left(V_{m+1}^{\prime}, P_{m+1}^{\prime}\right) \cdot \bar{\tau}_{\alpha}=0, \quad \alpha=1,2, \\
& \left.V_{m+1}^{\prime}\right|_{t=k T}=0 .
\end{aligned}
$$

To show convergence we divide the interval $[k T,(k+1) T]$ into $n$ subintervals with the length $\Delta t=\frac{T}{n}$, which will be chosen sufficiently small. Having proved the existence in the interval $[k T, k T+\Delta t]$ we obtain instead of the last equation in (4.25) the condition $\left.V_{m+1}^{\prime}\right|_{t=k T+\Delta t}=0$. Then considerations from the interval $[k T, k T+\Delta t]$ can be repeated in intervals $[k T+(s-1) \Delta t, k T+s \Delta t], s \leq n$. Hence we shall restrict our considerations to the interval $[k T, k T+\Delta t]$ only.

Multiplying $(4.25)_{1}$ by $V_{m+1}^{\prime}$ and integrating over $\Omega$ yields

$$
\frac{1}{2} \frac{d}{d t}\left|V_{m+1}^{\prime}\right|_{2, \Omega}^{2}+\int_{\Omega} V_{m}^{\prime} \cdot \nabla v_{m}^{\prime} \cdot V_{m+1}^{\prime} d x+\int_{\Omega} V_{m+1}^{\prime} \cdot \nabla v_{a} \cdot V_{m+1}^{\prime} d x+\nu\left\|V_{m+1}^{\prime}\right\|_{1, \Omega}^{2}=0 .
$$

By the Hölder and Young inequalities we have

$$
\frac{d}{d t}\left|V_{m+1}^{\prime}\right|_{2, \Omega}^{2}+\nu\left\|V_{m+1}^{\prime}\right\|_{1, \Omega}^{2} \leq c\left|\nabla v_{a}\right|_{3, \Omega}^{2}\left|V_{m+1}^{\prime}\right|_{2, \Omega}^{2}+c\left|\nabla v_{m}^{\prime}\right|_{2, \Omega}^{2}\left|V_{m}^{\prime}\right|_{3, \Omega}^{2}
$$

Integrating with respect to time implies

$$
\begin{aligned}
& \left|V_{m+1}^{\prime}(t)\right|_{2, \Omega}^{2}+\nu \int_{k T}^{k T+\Delta t}\left\|V_{m+1}^{\prime}\left(t^{\prime}\right)\right\|_{1, \Omega}^{2} d t^{\prime} \\
& \leq c \exp \left(\int_{k T}^{k T+\Delta t}\left|\nabla v_{a}\left(t^{\prime}\right)\right|_{3, \Omega}^{2} d t^{\prime}\right) \int_{k T}^{k T+\Delta t}\left|\nabla v_{m}^{\prime}\right|_{2, \Omega}^{2}\left\|V_{m}^{\prime}\right\|_{1, \Omega}^{2} d t^{\prime} \\
& \leq c e^{c A_{6}^{2}} \sup _{t^{\prime}}\left\|v_{m}^{\prime}\left(t^{\prime}\right)\right\|_{1, \Omega}^{2} \sup _{t^{\prime}}\left\|V_{m}^{\prime}\left(t^{\prime}\right)\right\|_{1, \Omega}^{2} \Delta t \\
& \leq c e^{c A_{6}^{2}} A^{2}(k)\left\|V_{m}^{\prime}\right\|_{2, \Omega \times(k T, k T+\Delta t)}^{2} \Delta t .
\end{aligned}
$$

From (4.25) we have also

$$
\left\|V_{m+1}^{\prime}\right\|_{2, \Omega \times(k T, k T+\Delta t)} \leq c\left(\left|v_{m}^{\prime} \cdot \nabla V_{m+1}^{\prime}\right|_{2, \Omega \times(k T, k T+\Delta t)}\right.
$$




$$
\begin{aligned}
& +\left|V_{m}^{\prime} \cdot \nabla v_{m}^{\prime}\right|_{2, \Omega \times(k T, k T+\Delta t)}+\left|V_{m+1}^{\prime} \cdot \nabla v_{a}\right|_{2, \Omega \times(k T, k T+\Delta t)} \\
& \left.+\left|v_{a} \cdot \nabla V_{m+1}^{\prime}\right|_{2, \Omega \times(k T, k T+\Delta t)}\right) .
\end{aligned}
$$

Now we estimate the particular terms on the r.h.s. of (4.27). We bound the first term by

$$
\begin{aligned}
& \int_{k T}^{k T+\Delta t} d t\left|v_{m}^{\prime} \cdot \nabla V_{m+1}^{\prime}\right|_{2, \Omega}^{2} \leq \int_{k T}^{k T+\Delta t} d t\left|v_{m}^{\prime}\right|_{4, \Omega}^{2}\left|\nabla V_{m+1}^{\prime}\right|_{4, \Omega}^{2} \\
& \leq \sup _{t}\left|v_{m}^{\prime}\right|_{4, \Omega}^{2}\left(\varepsilon_{1} \int_{k T}^{k T+\Delta t}\left|V_{m+1, x x}^{\prime}\right|_{2, \Omega}^{2} d t+c\left(1 / \varepsilon_{1}\right) \int_{k T}^{k T+\Delta t}\left|V_{m+1}^{\prime}\right|_{2, \Omega}^{2} d t\right) \\
& \leq c A^{2}\left(\varepsilon_{1}\left|V_{m+1, x x}^{\prime}\right|_{2, \Omega \times(k T, k T+\Delta t)}^{2}+c\left(1 / \varepsilon_{1}\right)\left|V_{m+1}^{\prime}\right|_{2, \Omega \times(k T, k T+\Delta t)}^{2}\right),
\end{aligned}
$$

the second by

$$
\begin{aligned}
& \int_{k T}^{k T+\Delta t} d t\left|V_{m}^{\prime}\right|_{6, \Omega}^{2}\left|\nabla v_{m}^{\prime}\right|_{3, \Omega}^{2} \leq \sup _{t}\left\|V_{m}^{\prime}\right\|_{1, \Omega}^{2} \int_{k T}^{k T+\Delta t} d t\left|\nabla v_{m}^{\prime}\right|_{3, \Omega}^{2} \\
& \leq \sup _{t}\left\|V_{m}^{\prime}\right\|_{1, \Omega}^{2}(\Delta t)^{1 / 2}\left(\int_{k T}^{k T+\Delta t} d t\left|\nabla v_{m}^{\prime}\right|_{3, \Omega}^{4}\right)^{1 / 2} \\
& \leq c A^{2}(\Delta t)^{1 / 2}\left\|V_{m}^{\prime}\right\|_{2, \Omega \times(k T, k T+\Delta t)}^{2},
\end{aligned}
$$

the third by

$$
\begin{aligned}
& \int_{k T}^{k T+\Delta t} d t\left|\nabla v_{a}\right|_{2, \Omega}^{2}\left|V_{m+1}^{\prime}\right|_{\infty, \Omega}^{2} \leq \sup _{t}\left|\nabla v_{a}\right|_{2, \Omega}^{2}\left(\varepsilon_{2}\left|V_{m+1, x x}^{\prime}\right|_{2, \Omega \times(k T, k T+\Delta t)}^{2}\right. \\
& \left.+c\left(1 / \varepsilon_{2}\right)\left|V_{m+1}^{\prime}\right|_{2, \Omega \times(k T, k T+\Delta t)}^{2}\right) \\
& \leq c A_{6}^{2}\left(\varepsilon_{2}\left|V_{m+1, x x}^{\prime}\right|_{2, \Omega \times(k T, k T+\Delta t)}^{2}+c\left(1 / \varepsilon_{2}\right)\left|V_{m+1}^{\prime}\right|_{2, \Omega \times(k T, k T+\Delta t)}^{2}\right) .
\end{aligned}
$$

Finally the last term on the r.h.s. of (4.27) is estimated by

$$
\begin{aligned}
& \int_{k T}^{k T+\Delta t} d t\left|v_{a}\right|_{4, \Omega}^{2}\left|\nabla V_{m+1}^{\prime}\right|_{4, \Omega}^{2} \leq \sup _{t}\left|v_{a}\right|_{4, \Omega}^{2} \int_{k T}^{k T+\Delta t} d t\left|\nabla V_{m+1}^{\prime}\right|_{4, \Omega}^{2} \\
& \leq c A_{6}^{2}\left(\varepsilon_{3}\left|V_{m+1, x x}^{\prime}\right|_{2, \Omega \times(k T, k T+\Delta t)}^{2}+c\left(1 / \varepsilon_{3}\right)\left|V_{m+1}^{\prime}\right|_{2, \Omega \times(k T, k T+\Delta t)}^{2}\right) .
\end{aligned}
$$

Utilizing the above estimates in (4.27), assuming that $\varepsilon_{1}-\varepsilon_{3}$ are sufficiently small and using (4.26) we obtain

$$
\left\|V_{m+1}^{\prime}\right\|_{2, \Omega \times(k T, k T+\Delta t)} \leq \varphi\left(A_{6}, A\right)(\Delta t)^{1 / 2}\left\|V_{m}^{\prime}\right\|_{2, \Omega \times(k T, k T+\Delta t)}
$$

where $\varphi$ is an increasing positive function. Hence we have convergence. This concludes the proof.

Therefore, we have proved local existence of solutions to problem (1.9) in the interval $[k T,(k+1) T]$, where $T$ is finite and fixed. To prove global existence we have to show that $\left\|v^{\prime}(k T)\right\|_{1, \Omega}$ can be estimated by a quantity independent of $k$. Then by (4.19) $A(k)$ can be chosen independently of $k$ too. Hence we need 
Lemma 4.3. Assume that $G_{1}(T)$ and $G_{2}(T)=A(0)+\|v(0)\|_{1, \Omega}$ are sufficiently small. Assume that

$$
v_{a} \in L_{2}\left(k T,(k+1) T ; H^{2}(\Omega)\right) \cap L_{4}\left(k T,(k+1) T ; H^{1}(\Omega)\right), \quad k \in \mathbb{N} .
$$

Then

$$
\left\|v^{\prime}(k T)\right\|_{1, \Omega} \leq\left\|v^{\prime}(0)\right\|_{1, \Omega}
$$

Proof. Multiplying (1.9) by $\operatorname{div} \mathbb{D}\left(v^{\prime}\right)$ and integrating over $\Omega$ implies

$$
\begin{aligned}
& \int_{\Omega} v_{t}^{\prime} \cdot \operatorname{div} \mathbb{D}\left(v^{\prime}\right) d x-\int_{\Omega}\left|\operatorname{div} \mathbb{D}\left(v^{\prime}\right)\right|^{2} d x+\int_{\Omega} v^{\prime} \cdot \nabla v^{\prime} \cdot \operatorname{div} \mathbb{D}\left(v^{\prime}\right) d x \\
& +\int_{\Omega} v^{\prime} \cdot \nabla v_{a} \cdot \operatorname{div} \mathbb{D}\left(v^{\prime}\right) d x+\int_{\Omega} v_{a} \cdot \nabla v^{\prime} \cdot \operatorname{div} \mathbb{D}\left(v^{\prime}\right) d x \\
& =\int_{\Omega} f^{\prime} \cdot \operatorname{div} \mathbb{D}\left(v^{\prime}\right) d x .
\end{aligned}
$$

The first term in (4.30) equals

$$
\begin{aligned}
& \int_{\Omega} \operatorname{div}\left(v_{t}^{\prime} \cdot \mathbb{D}\left(v^{\prime}\right)\right) d x-\int_{\Omega} \nabla v_{t}^{\prime} \cdot \mathbb{D}\left(v^{\prime}\right) d x=\int_{S} v_{t}^{\prime} \cdot \bar{n} \cdot \mathbb{D}\left(v^{\prime}\right) d S \\
& -\int_{\Omega} \nabla v_{t}^{\prime} \cdot \mathbb{D}\left(v^{\prime}\right) d x=\int_{S}\left(v_{n t}^{\prime} \bar{n}+v_{\tau_{\alpha}}^{\prime} \bar{\tau}_{\alpha}\right) \cdot \bar{n} \cdot \mathbb{D}\left(v^{\prime}\right) d S \\
& -\frac{1}{2} \frac{d}{d t} \int_{\Omega}\left|\mathbb{D}\left(v^{\prime}\right)\right|^{2} d x=-\frac{1}{2} \frac{d}{d t} \int_{\Omega}\left|\mathbb{D}\left(v^{\prime}\right)\right|^{2} d x,
\end{aligned}
$$

where the boundary conditions were used.

To continue considerations we have to examine the following elliptic problems:

$$
\begin{aligned}
& \operatorname{div} \mathbb{D}(v)=f, \\
& \left.v \cdot \bar{n}\right|_{S}=0, \\
& \left.\bar{n} \cdot \mathbb{D}(v) \cdot \bar{\tau}_{\alpha}\right|_{S}=0, \quad \alpha=1,2,
\end{aligned}
$$

and

$$
\begin{aligned}
& \operatorname{div} \mathbb{T}(v, p)=f \\
& \operatorname{div} v=0, \\
& \left.v \cdot \bar{n}\right|_{S}=0, \\
& \left.\bar{n} \cdot \mathbb{T}(v, p) \cdot \bar{\tau}_{\alpha}\right|_{S}=0, \quad \alpha=1,2 .
\end{aligned}
$$

Since the function $\eta=b \times x$, where $b$ is any constant vector, belongs to the kernels of operators (4.31) and (4.32), we see that $\int_{\Omega} r v_{\varphi} d x \eta$ belongs also to the kernels. Hence we have the following estimates for solutions of problems (4.31) and (4.32):

$$
\|v\|_{2, \Omega} \leq c\left(|f|_{2, \Omega}+|v|_{2, \Omega}\right),
$$

and

$$
\|v\|_{2, \Omega}+\left|p_{x}\right|_{2, \Omega} \leq c\left(|f|_{2, \Omega}+|v|_{2, \Omega}\right) .
$$


By the Hölder and interpolation inequalities (see [1, Ch. 3, Sec. 15]) we estimate the third term on the l.h.s. of (4.30) by

$$
\begin{aligned}
& c\left|v^{\prime}\right|_{6, \Omega}\left|v_{, x}^{\prime}\right|_{3, \Omega}\left|\operatorname{div} \mathbb{D}\left(v^{\prime}\right)\right|_{2, \Omega} \\
& \leq c\left|v^{\prime}\right|_{6, \Omega}\left(\left|v_{, x}^{\prime}\right|_{2, \Omega}^{1 / 2}\left\|v^{\prime}\right\|_{2, \Omega}^{1 / 2}+\left|v_{, x}^{\prime}\right|_{2, \Omega}\right)\left|\operatorname{div} \mathbb{D}\left(v^{\prime}\right)\right|_{2, \Omega} \\
& \leq c\left\|v^{\prime}\right\|_{1, \Omega}^{3 / 2}\left(\left|\operatorname{div} \mathbb{D}\left(v^{\prime}\right)\right|_{2, \Omega}^{1 / 2}+\left|v^{\prime}\right|_{2, \Omega}^{1 / 2}\right)\left|\operatorname{div} \mathbb{D}\left(v^{\prime}\right)\right|_{2, \Omega}+c\left\|v^{\prime}\right\|_{1, \Omega}^{2}\left|\operatorname{div} \mathbb{D}\left(v^{\prime}\right)\right|_{2, \Omega} \\
& \leq \varepsilon\left|\operatorname{div} \mathbb{D}\left(v^{\prime}\right)\right|_{2, \Omega}^{2}+c(1 / \varepsilon)\left(\left\|v^{\prime}\right\|_{1, \Omega}^{6}+\left\|v^{\prime}\right\|_{1, \Omega}^{4}\right),
\end{aligned}
$$

the fourth term by

$$
\begin{aligned}
& c\left|v^{\prime}\right|_{\infty, \Omega}\left|\nabla v_{a}\right|_{2, \Omega}\left|\operatorname{div} \mathbb{D}\left(v^{\prime}\right)\right|_{2, \Omega} \leq c\left|\nabla v_{a}\right|_{2, \Omega}\left(\left|v^{\prime}\right|_{6, \Omega}^{1 / 2}\left\|v^{\prime}\right\|_{2, \Omega}^{1 / 2}+\left|v^{\prime}\right|_{2, \Omega}\right)\left|\operatorname{div} \mathbb{D}\left(v^{\prime}\right)\right|_{2, \Omega} \\
& \leq c\left|\nabla v_{a}\right|_{2, \Omega}\left\|v^{\prime}\right\|_{1, \Omega}^{1 / 2}\left(\left|\operatorname{div} \mathbb{D}\left(v^{\prime}\right)\right|_{2, \Omega}^{1 / 2}+\left\|v^{\prime}\right\|_{1, \Omega}^{1 / 2}\right)\left|\operatorname{div} \mathbb{D}\left(v^{\prime}\right)\right|_{2, \Omega} \\
& \leq \varepsilon\left|\operatorname{div} \mathbb{D}\left(v^{\prime}\right)\right|_{2, \Omega}^{2}+c(1 / \varepsilon)\left(\left|\nabla v_{a}\right|_{2, \Omega}^{4}\left\|v^{\prime}\right\|_{1, \Omega}^{2}+\left|\nabla v_{a}\right|_{2, \Omega}^{2}\left\|v^{\prime}\right\|_{1, \Omega}^{2}\right),
\end{aligned}
$$

where the interpolation inequality is taken from [1, Ch. 3, Sec. 15], and finally, the fifth term by

$$
\begin{aligned}
& \left|v_{a}\right|_{6, \Omega}\left|v_{, x}^{\prime}\right|_{3, \Omega}\left|\operatorname{div} \mathbb{D}\left(v^{\prime}\right)\right|_{2, \Omega} \leq c\left\|v_{a}\right\|_{1, \Omega}\left(\left|v_{, x}^{\prime}\right|_{2, \Omega}^{1 / 2}\left\|v_{, x}^{\prime}\right\|_{1, \Omega}^{1 / 2}+\left|v_{, x}^{\prime}\right|_{2, \Omega}\right)\left|\operatorname{div} \mathbb{D}\left(v^{\prime}\right)\right|_{2, \Omega} \\
& \leq c\left\|v_{a}\right\|_{1, \Omega}\left\|v^{\prime}\right\|_{1, \Omega}^{1 / 2}\left(\left|\operatorname{div} \mathbb{D}\left(v^{\prime}\right)\right|_{2, \Omega}^{1 / 2}+\left\|v^{\prime}\right\|_{1, \Omega}^{1 / 2}\right)\left|\operatorname{div} \mathbb{D}\left(v^{\prime}\right)\right|_{2, \Omega} \\
& \leq \varepsilon\left|\operatorname{div} \mathbb{D}\left(v^{\prime}\right)\right|_{2, \Omega}^{2}+c(1 / \varepsilon)\left(\left\|v_{a}\right\|_{1, \Omega}^{4}+\left\|v_{a}\right\|_{1, \Omega}^{2}\right)\left\|v^{\prime}\right\|_{1, \Omega}^{2} .
\end{aligned}
$$

In view of the above considerations (4.30) implies

$$
\begin{aligned}
& \frac{d}{d t}\left|\mathbb{D}\left(v^{\prime}\right)\right|_{2, \Omega}^{2}+\nu\left|\operatorname{div} \mathbb{D}\left(v^{\prime}\right)\right|_{2, \Omega}^{2} \leq c\left(1+\left\|v^{\prime}\right\|_{1, \Omega}^{2}\right)\left\|v^{\prime}\right\|_{1, \Omega}^{4} \\
& +c\left(\left\|v_{a}\right\|_{1, \Omega}^{2}+\left\|v_{a}\right\|_{1, \Omega}^{4}\right)\left\|v^{\prime}\right\|_{1, \Omega}^{2}+c\left|f^{\prime}\right|_{2, \Omega}^{2} .
\end{aligned}
$$

From (4.5) and (4.35) we have

$$
\begin{aligned}
& \frac{d}{d t}\left(\left|v^{\prime}\right|_{2, \Omega}^{2}+\left|\mathbb{D}\left(v^{\prime}\right)\right|_{2, \Omega}^{2}\right)+\nu\left(\left|v^{\prime}\right|_{2, \Omega}^{2}+\left|\mathbb{D}\left(v^{\prime}\right)\right|_{2, \Omega}^{2}\right) \\
& \leq c\left(\left\|v_{a}\right\|_{1, \Omega}^{2}+\left\|v_{a}\right\|_{1, \Omega}^{4}+\left|\nabla v_{a}\right|_{3, \Omega}^{2}\right)\left(\left|v^{\prime}\right|_{2, \Omega}^{2}+\left|\mathbb{D}\left(v^{\prime}\right)\right|_{2, \Omega}^{2}\right) \\
& +c\left(1+\left\|v^{\prime}\right\|_{1, \Omega}^{2}\right)\left\|v^{\prime}\right\|_{1, \Omega}^{4}+c\left|f^{\prime}\right|_{2, \Omega}^{2} \\
& +c\left|\int_{\Omega} r v_{\varphi}^{\prime}(0) d x\right|^{2}+c\left|\int_{0}^{t} d t^{\prime} \int_{\Omega} r f_{\varphi}^{\prime} d x\right|^{2} .
\end{aligned}
$$

Let us introduce the notation

$$
\begin{aligned}
& X(t)=\left|v^{\prime}(t)\right|_{2, \Omega}^{2}+\left|\mathbb{D}\left(v^{\prime}(t)\right)\right|_{2, \Omega}^{2}, \\
& \beta(t)=\left\|v_{a}(t)\right\|_{1, \Omega}^{2}+\left\|v_{a}(t)\right\|_{1, \Omega}^{4}+\left|\nabla v_{a}(t)\right|_{3, \Omega}^{2} .
\end{aligned}
$$

Then (4.36) implies

$$
\begin{aligned}
& \frac{d}{d t}\left(X(t) e^{\nu t-c \int_{0}^{t} \beta\left(t^{\prime}\right) d t^{\prime}}\right) \leq c\left[\left(1+\left\|v^{\prime}\right\|_{1, \Omega}^{2}\right)\left\|v^{\prime}\right\|_{1, \Omega}^{4}\right. \\
& \left.+\left|f^{\prime}\right|_{2, \Omega}^{2}+\left|\int_{\Omega} r v_{\varphi}^{\prime}(0) d x\right|^{2}+\left|\int_{0}^{t} d t^{\prime} \int_{\Omega} r f_{\varphi}^{\prime}\left(t^{\prime}\right) d x\right|^{2}\right] e^{\nu t-c \int_{0}^{t} \beta\left(t^{\prime}\right) d t^{\prime}} .
\end{aligned}
$$


Integrating (4.37) with respect to time from $k T$ to $t \in(k T,(k+1) T]$ yields

$$
\begin{aligned}
& X(t) \leq e^{-\nu t+c \int_{k T}^{t} \beta\left(t^{\prime}\right) d t^{\prime}} \int_{k T}^{t}\left[\left(1+\left\|v^{\prime}\left(t^{\prime}\right)\right\|_{1, \Omega}^{2}\right)\left\|v^{\prime}\left(t^{\prime}\right)\right\|_{1, \Omega}^{4}\right. \\
& \left.+\left|f^{\prime}\left(t^{\prime}\right)\right|_{2, \Omega}^{2}\right] e^{\nu t^{\prime}} d t^{\prime}+c e^{c \int_{k T}^{t} \beta\left(t^{\prime}\right) d t^{\prime}}\left|\int_{\Omega} r v_{\varphi}^{\prime}(k T) d x\right|^{2} \\
& +e^{-\nu t+c \int_{k T}^{t} \beta\left(t^{\prime}\right) d t^{\prime}} c \int_{k T}^{t} d t^{\prime}\left|\int_{k T}^{t^{\prime}} d t^{\prime \prime} \int_{\Omega} r f_{\varphi}^{\prime}\left(t^{\prime \prime}\right) d x\right|^{2} e^{\nu t^{\prime}} \\
& +e^{-\nu(t-k T)+c \int_{k T}^{t} \beta\left(t^{\prime}\right) d t^{\prime}} X(k T) .
\end{aligned}
$$

Let us introduce the quantities

$$
\begin{aligned}
B(T)= & \sup _{k} \int_{k T}^{(k+1) T} \beta(t) d t \\
H(T)= & \sup _{k}\left[\int_{k T}^{(k+1) T}\left|f^{\prime}(t)\right|_{2, \Omega}^{2} d t+\left|\int_{\Omega} r v_{\varphi}^{\prime}(k T) d x\right|^{2}\right. \\
& \left.+\int_{k T}^{(k+1) T} d t\left|\int_{k T}^{t} d t^{\prime} \int_{\Omega} r f_{\varphi}^{\prime}\left(t^{\prime}\right) d x\right|^{2}\right] .
\end{aligned}
$$

Inserting $t=(k+1) T$ into (4.38) and using that

$$
\sup _{t \in[k T,(k+1) T]}\left\|v^{\prime}(t)\right\|_{1, \Omega} \leq A(k)+\left\|v^{\prime}(k T)\right\|_{1, \Omega} \equiv G_{2}(k, T)
$$

we obtain from (4.38) the inequality

$$
X((k+1) T) \leq e^{c B(T)}\left(G_{2}^{4}(k, T)+H(T)\right)+e^{-\nu T+c B(T)} X(k T) .
$$

Assuming that $X(k T), G_{2}(k, T), H(T)$ are sufficiently small and $T$ is sufficiently large we are able to show that

$$
X((k+1) T) \leq X(k T) .
$$

Starting from $k=0$ we can show step by step by applying Lemmas 4.1 and 4.2 that $G_{2}(k, T)$ can be chosen independent of $k$ and then

$$
X(k T) \leq X(0) \quad \text { for any } k \in \mathbb{N} .
$$

Hence (4.29) holds. This concludes the proof.

Lemmas 4.1-4.3 imply Theorem 2.

\section{References}

[1] O. V. Besov, V. P. Il'in and S. M. Nikol'skii, Integral Representation of Functions and Theorems of Imbedding, Nauka, Moscow, 1975 (in Russian). 
[2] V. P. Kochin, I. A. Kibel and N. V. Rose, Theoretical Hydrodynamics, Moscow, 1963 (in Russian).

[3] O. A. Ladyzhenskaya, On unique global solvability of three-dimensional Cauchy problem for the Navier-Stokes equations under the axial symmetry, Zap. Nauchn. Sem. LOMI 7 (1968) 155-177 (in Russian).

[4] L. Landau and E. Lifshitz, Hydrodynamics, Nauka, Moscow, 1986 (in Russian).

[5] P. B. Mucha, Stability of nontrivial solutions of the Navier-Stokes system on the threedimensional torus, J. Diff. Equs. 172 (2001), 359-375.

[6] G. Ponce, R. Racke, T. C. Sideris and E. S. Titi, Global stability of large solutions to the 3d Navier-Stokes equations, Comm. Math. Phys. 159 (1994), 329-341.

[7] M. R. Ukhovskij and V. I. Yudovich, Axially symmetric motions of ideal and viscous fluids filling all space, Prikl. Mat. Mekh. 32 (1968) 59-69 (in Russian).

[8] W. M. Zajączkowski, Global special regular solutions to Navier-Stokes equations in a cylindrical domain and with boundary slip conditions, Gakuto Series in Math. Vol. 21 (2004), $1-188$. 